\title{
Intermediate statistics for a system with symplectic symmetry: the Dirac rose graph
}

\author{
J.M. Harrison ${ }^{1}$ and B. Winn ${ }^{2}$ \\ ${ }^{1}$ Department of Mathematics, Baylor University, One Bear Place, Waco, Texas 76798, U.S.A. \\ ${ }^{2}$ Department of Mathematical Sciences, Loughborough University, Loughborough, LE11 3TU, \\ U.K.
}

$3^{\text {rd }}$ July 2012

\begin{abstract}
We study the spectral statistics of the Dirac operator on a rose-shaped grapha graph with a single vertex and all bonds connected at both ends to the vertex. We formulate a secular equation that generically determines the eigenvalues of the Dirac rose graph, which is seen to generalise the secular equation for a star graph with Neumann boundary conditions. We derive approximations to the spectral pair correlation function at large and small values of spectral spacings, in the limit as the number of bonds approaches infinity, and compare these predictions with results of numerical calculations. Our results represent the first example of intermediate statistics from the symplectic symmetry class.
\end{abstract}

\section{Introduction}

For classically chaotic quantum systems with an anti-unitary symmetry (for example, time reversibility), the spectral statistics are conjectured to be of random-matrix type from the Gaußian Orthogonal Ensemble [1,2]. If the motion is generated by a Hamiltonian of a half-integer spin particle, then instead the statistics of the Gaußian Symplectic Ensemble are expected [3, 4]. This is a strong form of universality, where a single exact expression for each spectral statistic is shared by all members of the universality class.

Between the dynamical extremes of full chaos, and complete integrability, one can find quantum systems with a variety of intermediate spectral statistics. Systems with intermediate statistics exhibit properties such as linear level repulsion and an exponential decay in the probability for large spacings. However, as was pointed out in [5] they do not share precisely the same spectral statistics.

Prominent examples that have been investigated in recent years include [6] the Anderson model at the metal-insulator transition point, [7, 8, 9] Aharonov-Bohm integrable billiards, $[10,11,12]$ rectangular billiards, or other integrable dynamical system, perturbed by the addition of a point singularity (this example has often been called the Šeba billiard) and $[13,14,15]$ several other examples such as polygonal billiards with rational angles. (In [16] an alternative construction of the operator corresponding to the Šeba billiard is given which exhibits Poissonian spectral statistics.)

Some other situations in which intermediate spectral statistics appear include [17] the intermediate statistics of eigenphases of quantum maps, and [18] a one-dimensional model 
for intermediate statistics formed of a gas of energy levels interacting with a logarithmic potential.

Spectral statistics of Šeba billiards (see also [19, 20]) have been thoroughly investigated in $[21,22,23,24,25,26]$. This model is particularly note-worthy since it constitutes an example of a member of a mini universality class of integrable systems perturbed by a rank-one singularity. Belonging to this class are quantum Neumann star graphs [27, 28], Šeba billiards and quantum Neumann rose graphs (see section 2.2 below).

As a measure of the spectral correlations we will mainly be using the pair correlation function $R_{2}(x)$ [29]. For a spectrum $\left\{\lambda_{n}\right\}$, scaled so that the mean spacing is 1 , we define $R_{2}(x)$ by

$$
\lim _{N \rightarrow \infty} \frac{1}{N} \sum_{m=0}^{N} \sum_{n=0}^{N} g\left(\lambda_{n}-\lambda_{m}\right)=g(0)+\int_{-\infty}^{\infty} g(x) R_{2}(x) \mathrm{d} x,
$$

where $g$ belongs to an appropriate class of test functions. The first term on the right-hand side of (1) comes from the diagonal terms $m=n$. By considering $g$ to be approximately an indicator function we see that $R_{2}(x)$ is a measure of pairs of levels (regardless of ordering) that lie within a given distance of each other. Note that unlike the nearest-neighbour spacing statistic that is sometimes studied, $R_{2}(x)$ is not a probability density.

The spectral statistics that are shared by Neumann star graphs and Šeba billiards have been studied in some detail. The pair correlation function for small $x$ was studied in $[13,22]$ and it was observed that it behaves as

$$
R_{2}(x) \approx \frac{\pi \sqrt{3}}{2} x \quad \text { as } x \rightarrow 0 .
$$

For the large $x$ asymptotics, a full series expansion has been derived [27, 28]. Keeping terms up to $x^{-12}$ the expansion reads

$$
R_{2}(x)=1+\frac{2}{\pi^{2} x^{2}}+\frac{76}{\pi^{4} x^{4}}-\frac{1088}{\pi^{6} x^{6}}+\frac{9280}{\pi^{8} x^{8}}-\frac{64000}{\pi^{10} x^{10}}+\mathrm{O}\left(\frac{1}{x^{12}}\right), \quad \text { as } x \rightarrow \infty .
$$

These behaviours are illustrated in figure 1, in which they are compared to a numerical calculation of the pair-correlation function.

Our motivation is to analyse the spectral statistics of a system in the Šeba class, with a symplectic symmetry. The most convenient model to use for this investigation are quantum graphs, since it is known that quantum star graphs with Neumann boundary conditions have statistics in the Šeba class, and Bolte and Harrison [30, 31] successfully quantised quantum graphs with the Dirac operator, and showed that generically the statistics of the Gaußian symplectic ensemble are found.

The only obstacle to this programme is that the construction of [30] does not allow graphs with vertices of valency one, which is most of the vertices of a star graph. For this reason we re-attach the loose ends of the star graph to the central vertex, to form a graph which we call a rose graph (see figure 2). We will see (section 2.2) that despite this change in the graph topology important features of the spectral analysis for star graphs survive the transformation.

The article is laid out in the following way. In section 2 we introduce the general scheme used to quantise the rose graph with the Dirac operator, in particular we derive a simple secular equation whose roots provide the spectrum of the graph with a similar structure to the well known secular equation of the Neumann star graph. Section 3 presents the derivation of the small and large parameter asymptotics of the pair correlation function 


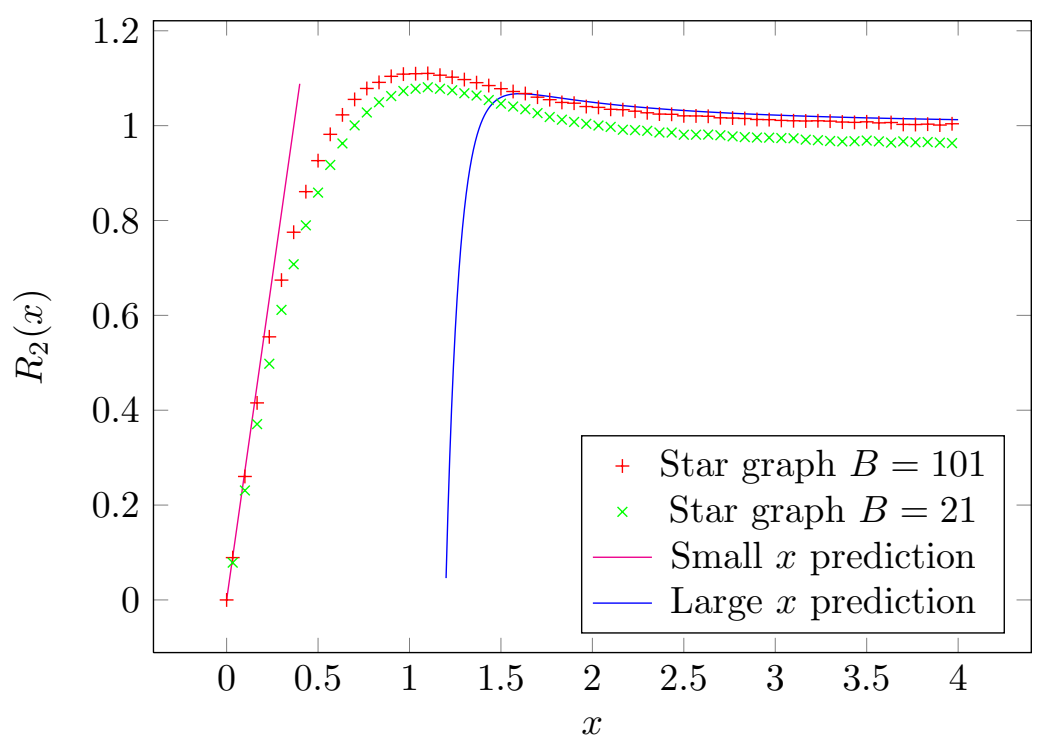

Figure 1: The pair correlation function numerically calculated for a $B=21$ bond (green) and $B=101$ bond (red) star graph with Neumann boundary conditions. Also plotted are the curves corresponding to the large and small values of the parameter, (3) and (2) respectively. The numerical plots are averaged over 100 realisations of the bond lengths, and 150000 eigenvalues were computed.

for the Dirac rose graph. Section 4 draws together the results and compares them with numerical calculations of the Dirac rose graph and a Neumann star graph.

\section{Quantisation of rose graphs with the Dirac operator}

A graph $\mathcal{G}$ consists of a set of vertices $\mathcal{V}$ with pairs of vertices connected by bonds, as in figure 2. Two vertices $u, v \in \mathcal{V}$ are adjacent $u \sim v$ if the pair $(u, v)$ is in the set of bonds $\mathcal{B}$, which may be associated with the set $\{1,2, \ldots, B\}$. We will also use $u \sim b$ and $v \sim b$ to show that the bond $b$ is connected to $u$ and $v$. Each bond $b$ is associated with an interval $\left[0, L_{b}\right]$ where $L_{b}$ is the length of $b . \mathscr{L}=2 \sum_{b=1}^{B} L_{b}$ will denote twice the total length of the graph; a natural measure of the volume of the graph as each bond can be traversed in two directions. We will assume each $L_{b}$ lies in an interval $\left[1-(2 B)^{-1}, 1+(2 B)^{-1}\right]$ and that the set of bond lengths are rationally independent. On $\left[0, L_{b}\right]$ we define a coordinate $x_{b}$ such that $x_{b}=0$ at the vertex o $(b)$ and $x_{b}=L_{b}$ at the vertex $\mathrm{t}(b) ; \mathrm{o}(b)$ and $\mathrm{t}(b)$ are called the origin and terminus of $b$ respectively. We will use $x$ to denote a general coordinate on the graph when the particular bond is not significant. The number of bonds $b$ with o $(b)=v$ or $\mathrm{t}(b)=v$ is $d_{v}$ the degree of the vertex $v$. Note that a bond $b$ with $\mathrm{o}(b)=\mathrm{t}(b)=v$, as seen in the rose graph, counts twice when determining the degree of $v$. It is sometimes convenient to have notation for the reverse of a bond; $\bar{b}$ is a bond with o $(\bar{b})=t(b)$ and $\mathrm{t}(\bar{b})=\mathrm{o}(b)\left(L_{\bar{b}}=L_{b}\right)$.

On an interval $\left[0, L_{b}\right]$ the time independent Dirac equation reads,

$$
-\mathrm{i} \alpha \frac{\mathrm{d} \boldsymbol{\psi}_{b}}{\mathrm{~d} x_{b}}+m \beta \boldsymbol{\psi}_{b}=E \boldsymbol{\psi}_{b}
$$



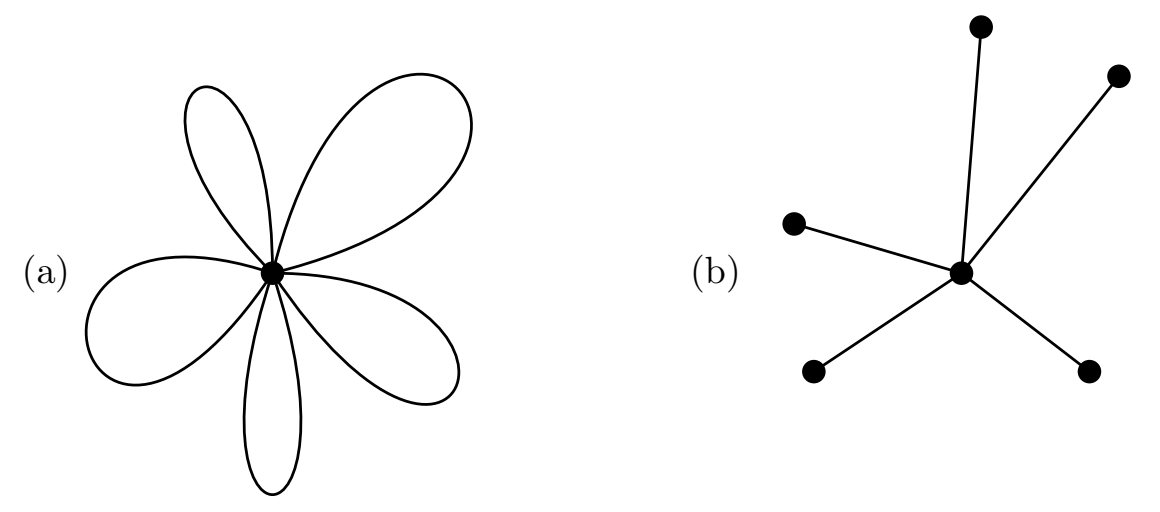

Figure 2: (a) A rose graph with 5 bonds; (b) a star graph with 5 bonds.

where $\alpha$ and $\beta$ satisfy the relations $\alpha^{2}=\beta^{2}=\mathrm{I}$ and $\alpha \beta+\beta \alpha=0$ which define the Dirac algebra in one dimension. The dimension of $\alpha$ and $\beta$ depends on the interpretation of the Dirac equation in one dimension. If one looks for the simplest faithful irreducible representation of the Dirac algebra $\alpha$ and $\beta$ will be $2 \times 2$ matrices acting on two component spinors. On the other hand, if one regards the equation in one dimension as the restriction to a wire of a Dirac equation in three dimensions it is natural to expect four component spinors. It turns out that these two different approaches lead to the same scattering problem [30]. In order to impose time-reversal symmetry with two component spinors it is necessary to work with pairs of bonds connecting vertices, effectively reintroducing four components to the wave function for each adjacent pair of vertices. To simplify the current presentation we will only consider four component spinors from the outset and fix

$$
\alpha=\left(\begin{array}{rrrr}
0 & 0 & 0 & -\mathrm{i} \\
0 & 0 & \mathrm{i} & 0 \\
0 & -\mathrm{i} & 0 & 0 \\
\mathrm{i} & 0 & 0 & 0
\end{array}\right) \quad \text { and } \quad \beta=\left(\begin{array}{rrrr}
1 & 0 & 0 & 0 \\
0 & 1 & 0 & 0 \\
0 & 0 & -1 & 0 \\
0 & 0 & 0 & -1
\end{array}\right)
$$

Given the operator on the collection of intervals we must now specify a domain for which it is self-adjoint. To do this we fix appropriate matching conditions between the spinors on the set of intervals where they meet at the vertices of the $\mathcal{G}$. Such matching conditions were classified in [30]. In particular there is a natural generalisation of matching conditions that define a self-adjoint Laplace operator on the graph to conditions for a Dirac operator.

For comparison the time-independent free Schrödinger equation on an interval reads,

$$
-\frac{\mathrm{d}^{2} \psi_{b}}{\mathrm{~d} x_{b}^{2}}=E \psi_{b}
$$

Matching conditions between the functions on the individual intervals where they meet at a vertex $v$ are expressed as $d_{v}$ linear relations amongst the values of the functions $\psi_{b}$ and their derivatives $\psi_{b}^{\prime}$ evaluated at the end of each interval connected to $v$. For example, a 
common choice of matching conditions for the Laplace operator are Neumann conditions; $\psi$ is continuous at each vertex $v$;

$$
\begin{aligned}
\psi_{b}(0) & =\gamma \text { for all } b \text { with } \mathrm{o}(b)=v, \\
\psi_{b}\left(L_{b}\right) & =\gamma \text { for all } b \text { with } \mathrm{t}(b)=v,
\end{aligned}
$$

(Note that $\gamma$ is not a fixed constant but rather a placeholder for the value of the wave function at the vertex $v$ whatever that happens to be) and the outgoing derivatives at $v$ sum to zero,

$$
\sum_{b \mid \mathrm{o}(b)=v} \psi_{b}^{\prime}(0)-\sum_{b \mid \mathrm{t}(b)=v} \psi_{b}^{\prime}\left(L_{b}\right)=0 .
$$

Fixing such matching conditions at each vertex of the graph ensures that the Laplace operator is self-adjoint. A full description of all matching conditions for which the operator is self-adjoint has been obtained in various forms [32, 33, 34], however for the current discussion an understanding of Neumann conditions is sufficient.

For the Laplace operator it is clear that a wave function restricted to bond $b$ can be written as a linear superposition of two plane waves,

$$
\psi_{b}\left(x_{b}\right)=Q_{b} \mathrm{e}^{\mathrm{i} k x_{b}}+R_{b} \mathrm{e}^{-\mathrm{i} k x_{b}},
$$

where $k^{2}=E$. For simplicity we can consider the centre of the star $v$ where $v=\mathrm{o}(b)$ for every $b \sim v$. Then $\mathbf{Q}=\left(Q_{1}, \ldots, Q_{B}\right)$ and $\mathbf{R}$, defined similarly, are vectors of the coefficients of incoming and outgoing plane wave solutions at $v$. Using the matching conditions these can be related by a unitary matrix $\sigma^{(v)}(k)$ the vertex scattering matrix,

$$
\mathbf{R}=\sigma^{(v)}(k) \mathbf{Q} .
$$

With Neumann conditions at $v$ the vertex scattering matrix takes a particularly simple form, see e.g. [35],

$$
\sigma_{b c}^{(v)}=\left\{\begin{array}{cc}
\frac{2}{d_{v}} & \text { if } b \neq c \\
\frac{2}{d_{v}}-1 & \text { if } b=c
\end{array} .\right.
$$

Components of $\sigma^{(v)}$ are scattering amplitudes relating an incoming plane wave on bond $c$ to an outgoing plane wave on bond $b$. Time-reversal symmetry implies that $\sigma^{(v)}=\left(\sigma^{(v)}\right)^{\mathrm{T}}$.

To quantise a graph with two component spinors one may treat the spinor matching conditions by analogy with those of the Laplace operator. The first component of the spinors evaluated at the ends of the bonds takes the place of the value of the scalar wave function of the Laplace operator while the derivative of the wave function evaluated at the ends of the intervals is replaced by the value of the second component of the spinor at the bond ends. With the four component spinors, necessary to incorporate time-reversal symmetry, the story is much the same. However, now a vector of the first and second components of the spinor is treated by analogy with values of the wave function and the third and fourth components replace values of the derivative. In the following we summarise the relevant results from [30] concerning the quantisation of a graph with the Dirac operator.

If $\boldsymbol{\psi}=\left(\psi_{1}, \ldots, \psi_{4}\right)^{\mathrm{T}}$ is a 4 -component spinor satisfying the Dirac equation (4) it can be expressed as a linear combination of four plane waves two traveling in each direction 
along the bond. For $E>m, \psi_{b}$ is of the form

$$
\begin{aligned}
\psi_{b}=Q_{b}\left(\begin{array}{c}
-\mathrm{i} \sqrt{E+m} \\
0 \\
0 \\
\sqrt{E-m}
\end{array}\right) \mathrm{e}^{\mathrm{i} k x_{b}}+P_{b}\left(\begin{array}{c}
0 \\
\sqrt{E+m} \\
-\mathrm{i} \sqrt{E-m} \\
0
\end{array}\right) \mathrm{e}^{\mathrm{i} k x_{b}} \\
+R_{b}\left(\begin{array}{c}
0 \\
\sqrt{E+m} \\
\mathrm{i} \sqrt{E-m} \\
0
\end{array}\right) \mathrm{e}^{-\mathrm{i} k x_{b}}+S_{b}\left(\begin{array}{c}
\mathrm{i} \sqrt{E+m} \\
0 \\
0 \\
\sqrt{E-m}
\end{array}\right) \mathrm{e}^{-\mathrm{i} k x_{b},}
\end{aligned}
$$

where $k^{2}=E^{2}-m^{2}$, and $P_{b}, Q_{b}, R_{b}, S_{b}$ are constants of integration. It is convenient to define

$$
\mathbf{Q}_{b}=\left(\begin{array}{r}
-\mathrm{i} Q_{b} \\
P_{b}
\end{array}\right) \quad \text { and } \quad \mathbf{R}_{b}=\left(\begin{array}{c}
\mathrm{i} S_{b} \\
R_{b}
\end{array}\right) .
$$

Then vectors of coefficients of the incoming and outgoing waves at the vertex of a star can be written as,

$$
\mathbf{Q}=\left(\mathbf{Q}_{1}^{\mathrm{T}}, \ldots, \mathbf{Q}_{B}^{\mathrm{T}}\right)^{\mathrm{T}} \quad \text { and } \quad \mathbf{R}=\left(\mathbf{R}_{1}^{\mathrm{T}}, \ldots, \mathbf{R}_{B}^{\mathrm{T}}\right)^{\mathrm{T}} .
$$

As in the scalar case, vectors of the incoming and outgoing coefficients can be related via a vertex scattering matrix;

$$
\mathbf{R}=\sigma^{(v)}(k) \mathbf{Q} .
$$

Time-reversal symmetry requires that

$$
\left(\sigma^{(v)}\right)^{\mathrm{T}}=J^{-1} \sigma^{(v)} J
$$

where $J$ is a block diagonal matrix

$$
J=\left(\begin{array}{cccc}
\left(\begin{array}{cc}
0 & 1 \\
-1 & 0
\end{array}\right) & & & \\
& & \ddots & \\
& & & \left(\begin{array}{cc}
0 & 1 \\
-1 & 0
\end{array}\right)
\end{array}\right)
$$

If the vertex scattering matrix is also divided into $2 \times 2$ blocks $\left(\sigma^{(v)}\right)^{b c}$, which relate pairs of incoming spinors on bond $b$ to outgoing pairs on bond $c$, the symmetry condition (17) reads

$$
\left(\sigma^{(v)}\right)^{c b}=\operatorname{det}\left(\left(\sigma^{(v)}\right)^{b c}\right)\left(\left(\sigma^{(v)}\right)^{b c}\right)^{-1} .
$$

We can factor a block of the vertex scattering matrix so

$$
\left(\sigma^{(v)}\right)^{b c}=X_{b c} u_{b c},
$$

where $u_{b c} \in \mathrm{SU}(2)$, the symmetry condition is then $X_{c b}=X_{b c}$ and $u_{c b}=u_{b c}^{-1}$. In this notation spinor scattering on the graph is defined by a scalar scattering amplitudes $X_{b c}$ which define the transition probability from bond $b$ to $c$, just as in the case of scalar wave functions, and an additional rotation between the spinors when passing through the vertex, $u_{b c}$. In general these transition amplitudes and spinor rotations are obtained 
from the matching conditions and will also satisfy the constraint that the whole scattering matrix $\sigma^{(v)}$ is unitary. One straightforward way to satisfy these symmetry relations also establishes a connection with the scattering matrices of the Schrödinger operator. A vertex scattering matrix of the form

$$
\sigma^{(v)}=U^{(v)}\left\{X^{(v)} \otimes \mathrm{I}_{2}\right\}\left(U^{(v)}\right)^{-1},
$$

where $X^{(v)}$ is a symmetric unitary $d_{v} \times d_{v}$ matrix and $U^{(v)}=\operatorname{diag}\left\{u_{1}, \ldots, u_{d_{v}}\right\}$ with $u_{b} \in \mathrm{SU}(2)$ satisfies the symmetry condition (17). The $d_{v}$ matrices $u_{b}$ can be thought of as defining a spinor rotation when leaving the vertex on bond $b$ or the inverse rotation when entering the vertex from that bond. A symmetric unitary $d_{v} \times d_{v}$ matrix of transition amplitudes $X^{(v)}$ is precisely the form of vertex scattering matrix obtained for the Laplace operator with time-reversal symmetry (12). In fact, given matching conditions that make the Laplace operator self-adjoint with a $d_{v} \times d_{v}$ vertex scattering matrix $X^{(v)}$ we can define analogous matching conditions which will make a Dirac operator self-adjoint for any given set of spinor rotations $\left\{u_{1}, \ldots, u_{d_{v}}\right\}$. For instance matching conditions analogous to the Neumann conditions defined for the Laplace operator (which with a slight abuse of terminology we will also call Neumann conditions) are

$$
\begin{aligned}
u_{\mathrm{o}(b)} \mathbf{v}_{b}(0) & =\boldsymbol{\gamma} \text { for all } b \text { with } \mathrm{o}(b)=v \\
u_{\mathrm{t}(b)} \mathbf{v}_{b}\left(L_{b}\right) & =\boldsymbol{\gamma} \text { for all } b \text { with } \mathrm{t}(b)=v
\end{aligned}
$$

where on each bond $b$ we have such a solution $\psi_{b}=\left(\psi_{b 1}, \ldots \psi_{b 4}\right)^{\mathrm{T}}$ to $(4)$ and we define

$$
\mathbf{v}_{b}(x)=\left(\begin{array}{c}
\psi_{b 1}(x) \\
\psi_{b 2}(x)
\end{array}\right) \quad \mathbf{w}_{b}(x)=\left(\begin{array}{c}
-\psi_{b 4}(x) \\
\psi_{b 3}(x)
\end{array}\right) .
$$

In (22)-(23) $\boldsymbol{\gamma}$ is again a placeholder for a common value of the spinor at the vertex rather than a fixed constant vector. In addition the Neumann condition on the derivatives (9) becomes,

$$
\sum_{b \mid \mathrm{o}(b)=v} u_{\mathrm{o}(b)} \mathbf{w}_{b}(0)-\sum_{b \mid \mathrm{t}(b)=v} u_{\mathrm{t}(b)} \mathbf{w}_{b}\left(L_{b}\right)=0 .
$$

Together applying these conditions at all vertices of a graph defines a self-adjoint realization of the Dirac operator [30, section 5].

To each bond $b$ of a graph we now see that there correspond a pair of $2 \times 2$ unitary matrices $u_{\mathrm{o}(b)}$ and $u_{\mathrm{t}(b)}$ which rotate the spinor when entering the vertices at the origin and terminus of $b$ respectively. At a vertex, during the transition from a bond $b_{j}$ to a bond $b_{i}$ the spinor is rotated according to the matrix $u_{b_{i} b_{j}} \in \mathrm{SU}(2)$ where

$$
u_{b_{i} b_{j}}:=u_{\mathrm{o}\left(b_{i}\right)}\left(u_{\mathrm{t}\left(b_{j}\right)}\right)^{-1},
$$

It will be convenient to associate spin matrices to bonds rather than vertices, so for this reason we define

$$
\tilde{w}_{b}:=u_{\mathrm{o}(b)}\left(u_{\mathrm{t}(b)}\right)^{-1}
$$

and

$$
w_{b}:=\left(u_{\mathrm{t}(b)}\right)^{-1} u_{\mathrm{o}(b)} .
$$


We note that if $\bar{b}$ is the reversal of the bond $b$,

$$
\begin{aligned}
w_{\bar{b}} & =\left(u_{\mathrm{t}(\bar{b})}\right)^{-1} u_{\mathrm{o}(\bar{b})} \\
& =\left(u_{\mathrm{o}(b)}\right)^{-1} u_{\mathrm{t}(b)} \\
& =w_{b}^{-1} .
\end{aligned}
$$

If the $2 B$ matrices $\left\{u_{\mathrm{o}(b)}, u_{\mathrm{t}(b)}\right\}_{b \in \mathcal{B}}$ are random and independently distributed, then the matrices $\left\{w_{b}\right\}_{b \in \mathcal{B}}$ are independently distributed. If the matrices $\left\{u_{\mathrm{o}(b)}, u_{\mathrm{t}(b)}\right\}_{b \in \mathcal{B}}$ are independently distributed with Haar measure on $\mathrm{SU}(2)$, then the $w_{b}$ are Haar-distributed too, and the angles $\left\{\theta_{b}\right\}_{b \in \mathcal{B}}$ defined on $[0, \pi]$ by

$$
\operatorname{Tr} \tilde{w}_{b}=\operatorname{Tr} w_{b}=: 2 \cos \theta_{b},
$$

are identically independently distributed with a sine-squared distribution:

$$
\mathbb{P}\left(\theta_{b}<x\right)=\frac{2}{\pi} \int_{0}^{x} \sin ^{2} \phi \mathrm{d} \phi, \quad 0 \leqslant x \leqslant \pi .
$$

The traces themselves have a semi-circle distribution:

$$
\mathbb{P}\left(\operatorname{Tr} w_{b}<x\right)=\frac{1}{2 \pi} \int_{-2}^{x} \sqrt{4-t^{2}} \mathrm{~d} t, \quad-2 \leqslant x \leqslant 2 .
$$

Bolte and Harrison proved a trace formula for the eigenvalues of the Dirac operator on an arbitrary graph in terms of periodic orbits [30]. In order to state the trace formula, we need to define a few quantities. A periodic orbit $p$ is a closed path on the graph, modulo cyclic shifts. So $p$ can be written as a sequence of connected bonds $p=\left(b_{1}, b_{2}, \ldots, b_{n}\right)$ where $\mathrm{t}\left(b_{j}\right)=\mathrm{o}\left(b_{j+1}\right)$ and $\mathrm{t}\left(b_{n}\right)=\mathrm{o}\left(b_{0}\right)$. We denote by the symbol $\mathcal{P}$ the family of all periodic orbits on the graph. It is possible that an orbit $p \in \mathcal{P}$ can be written as a repetition of a shorter periodic orbit, in which case $r_{p}$, the repetition number of $p$, is the maximal number of repetitions of a shorter orbit contained in $p$. $A_{p}$ will denote the product of all scattering amplitudes accumulated along the orbit. For the free Schrödinger operator this is simply be products of the elements of the vertex scattering matrices;

$$
A_{p}=\sigma_{b_{2} b_{1}}^{\left(\mathrm{t}\left(b_{1}\right)\right)} \sigma_{b_{3} b_{2}}^{\left(\mathrm{t}\left(b_{2}\right)\right)} \ldots \sigma_{b_{1} b_{n}}^{\left(\mathrm{t}\left(b_{n}\right)\right)} .
$$

While for the Dirac operator we replace $\sigma_{b_{j+1} b_{j}}^{\left(\mathrm{t}\left(b_{j}\right)\right)}$ with $X_{b_{j+1} b_{j}}^{\left(\mathrm{t}\left(b_{j}\right)\right)}$. We will denote by $d_{p}$ the matrix product of all spin matrices accumulated on the orbit:

$$
d_{p}:=u_{b_{n} b_{n-1}} u_{b_{n-1} b_{n-2}} \cdots u_{b_{2} b_{1}} u_{b_{1} b_{n}} \in \mathrm{SU}(2) .
$$

Because of the cyclic invariance of the trace, we have

$$
\operatorname{Tr} d_{p}=\operatorname{Tr}\left(w_{b_{n}} w_{b_{n-1}} \cdots w_{b_{2}} w_{b_{1}}\right) .
$$

$\ell_{p}$ denotes the length of the orbit (i.e. the sum of lengths of bonds over which the orbit passes).

Let $h$ be a test function whose Fourier transform $\hat{h}(\ell)=\int_{-\infty}^{\infty} h(k) \mathrm{e}^{-2 \pi \mathrm{i} k \ell} \mathrm{d} k$ is smooth and compactly supported. Then, in terms of quantities defined above, the trace formula reads:

$$
\sum_{n=1}^{\infty} h\left(k_{n}\right)=\frac{\mathscr{L}}{\pi} H(0)+\frac{2}{\pi} \sum_{p \in \mathcal{P}} \frac{\ell_{p}}{r_{p}}\left(\frac{\operatorname{Tr} d_{p}}{2}\right) A_{p} H\left(\ell_{p}\right)
$$


where

$$
H(\ell):=\int_{-\infty}^{\infty} h(k) \cos k \ell \mathrm{d} k .
$$

In (36) recall that the quantity $\mathscr{L}$ is twice the total length of the graph.

\subsection{Dirac rose graphs}

On the rose graph there are $B$ intervals with both ends of each interval connected to the single central vertex, figure 2(b). Eigenspinors on the bonds have the form shown in equation (13). The Neumann matching conditions at the central vertex, which will determine the constants of integration, are,

$$
u_{\mathrm{o}(b)} \mathbf{v}_{b}(0)=u_{\mathrm{t}(b)} \mathbf{v}_{b}\left(L_{b}\right)=\boldsymbol{\gamma} \quad \text { for all } b,
$$

which imposes continuity of the first pair of components of the spinor, and

$$
\sum_{b=1}^{B} u_{\mathrm{o}(b)} \mathbf{w}_{b}(0)=\sum_{b=1}^{B} u_{\mathrm{t}(b)} \mathbf{w}_{b}\left(L_{b}\right) .
$$

Using the solution (13) and (14), condition (38) reads

$$
u_{\mathrm{o}(b)}\left(\mathbf{Q}_{b}+\mathbf{R}_{b}\right)=u_{\mathrm{t}(b)}\left(\mathbf{Q}_{b} \mathrm{e}^{\mathrm{i} k L_{b}}+\mathbf{R}_{b} \mathrm{e}^{-\mathrm{i} k L_{b}}\right)=\frac{\gamma}{\sqrt{E+m}},
$$

which gives

$$
\left(u_{\mathrm{o}(b)}-u_{\mathrm{t}(b)} \mathrm{e}^{\mathrm{i} k L_{b}}\right) \mathbf{Q}_{b}=-\left(u_{\mathrm{o}(b)}-u_{\mathrm{t}(b)} \mathrm{e}^{-\mathrm{i} k L_{b}}\right) \mathbf{R}_{b} .
$$

From (40),

$$
\frac{u_{\mathrm{o}(b)}^{-1} \boldsymbol{\gamma}}{\sqrt{E+m}}=\mathbf{Q}_{b}+\mathbf{R}_{b}
$$

and so, eliminating $\mathbf{R}_{b}$ using (41) we get

$$
\mathbf{Q}_{b} \sin k L_{b}=\frac{1}{2 \mathrm{i} \sqrt{E+m}} u_{\mathrm{t}(b)}^{-1}\left(u_{\mathrm{o}(b)}-u_{\mathrm{t}(b)} \mathrm{e}^{-\mathrm{i} k L_{b}}\right) u_{\mathrm{o}(b)}^{-1} \gamma .
$$

Let us now, for simplicity, consider the case that $L_{b} k / \pi \notin \mathbb{Z}$ for every bond $b$. Applying the boundary condition (39) gives

$$
\sum_{b=1}^{B}-\mathrm{i} u_{\mathrm{o}(b)}\left(\mathbf{Q}_{b}-\mathbf{R}_{b}\right)=\sum_{b=1}^{B}-\mathrm{i} u_{\mathrm{t}(b)}\left(\mathbf{Q}_{b} \mathrm{e}^{\mathrm{i} k L_{b}}-\mathbf{R}_{b} \mathrm{e}^{-\mathrm{i} k L_{b}}\right),
$$

which simplifies to

$$
\sum_{b=1}^{B} 2\left(u_{\mathrm{o}(b)}-\mathrm{e}^{\mathrm{i} k L_{b}} u_{\mathrm{t}(b)}\right) \mathbf{Q}_{b}=\mathbf{0}
$$

upon inserting (41). Substituting (43), we get

$$
\begin{aligned}
& \frac{1}{\mathrm{i} \sqrt{E+m}} \sum_{b=1}^{B} \frac{1}{\sin k L_{b}}\left(\left(u_{\mathrm{o}(b)}-\mathrm{e}^{\mathrm{i} k L_{b}} u_{\mathrm{t}(b)}\right) u_{\mathrm{t}(b)}^{-1}\left(u_{\mathrm{o}(b)}-u_{\mathrm{t}(b)} \mathrm{e}^{-\mathrm{i} k L_{b}}\right) u_{\mathrm{o}(b)}^{-1}\right) \boldsymbol{\gamma}=\mathbf{0} \\
& \Rightarrow \sum_{b=1}^{B} \frac{1}{\sin k L_{b}}\left(u_{\mathrm{o}(b)} u_{\mathrm{t}(b)}^{-1}-\mathrm{e}^{\mathrm{i} k L_{b}} \mathrm{I}_{2}\right)\left(\mathrm{I}_{2}-u_{\mathrm{t}(b)} u_{\mathrm{o}(b)}^{-1} \mathrm{e}^{-\mathrm{i} k L_{b}}\right) \boldsymbol{\gamma}=\mathbf{0} \\
& \Rightarrow \quad \sum_{b=1}^{B} \frac{1}{\sin k L_{b}}\left(\tilde{w}_{b}+\tilde{w}_{b}^{-1}-2 \cos k L_{b} \mathrm{I}_{2}\right) \boldsymbol{\gamma}=\mathbf{0} .
\end{aligned}
$$


Since $\tilde{w}_{b}+\tilde{w}_{b}^{-1}=\operatorname{Tr} \tilde{w}_{b} \mathrm{I}_{2}=2 \cos \theta_{b} \mathrm{I}_{2}$, the condition for $k$ to be an eigenvalue becomes

$$
\sum_{b=1}^{B} \frac{\cos \theta_{b}-\cos k L_{b}}{\sin k L_{b}}=0 .
$$

Equation (47) is the secular equation for a Dirac rose graph.

Let us now consider what happens if $L_{b} k / \pi=n \in \mathbb{Z}$ for some bond $b$. In that case, (43) may be re-written as

$$
\begin{aligned}
\mathbf{0}=\mathbf{Q}_{b} \sin k L_{b} & =\frac{1}{2 \mathrm{i} \sqrt{E+m}} u_{\mathrm{t}(b)}^{-1}\left(u_{\mathrm{o}(b)}-(-1)^{n} u_{\mathrm{t}(b)}\right) u_{\mathrm{o}(b)}^{-1} \boldsymbol{\gamma} \\
& =\frac{1}{2 \mathrm{i} \sqrt{E+m}} u_{\mathrm{t}(b)}^{-1}\left(\tilde{w}_{b}-(-1)^{n} \mathrm{I}_{2}\right) u_{\mathrm{t}(b)} u_{\mathrm{o}(b)}^{-1} \boldsymbol{\gamma} .
\end{aligned}
$$

Thus, if $\tilde{w}_{b} \neq(-1)^{m} \mathrm{I}_{2}$, we find that $\boldsymbol{\gamma}=\mathbf{0}$, and consequently $\mathbf{Q}_{c}=\mathbf{R}_{c}=\mathbf{0}$ for all other $c \neq b$, since the rational independence of bond lengths means that $L_{c} k / \pi \notin \mathbb{Z}$. From the boundary condition (39), we then would have $\mathbf{Q}_{b}=\mathbf{R}_{b}=\mathbf{0}$, and there are consequently no non-trivial solutions to (4).

We therefore conclude that if all $\tilde{w}_{b} \neq \pm \mathrm{I}_{2}$, then the eigenvalues $k_{n}, n \in \mathbb{Z}$ are given by the condition (47), which is the central result of this paper. We also remark that if the matrices $\left\{u_{\mathrm{o}(b)}, u_{\mathrm{t}(b)}\right\}_{b \in \mathcal{B}}$ are chosen randomly independently with Haar measure, then almost-surely, $\tilde{w}_{b} \neq \pm \mathrm{I}_{2}$.

Kramer's degeneracy is evident in equation (46), since for any value of $k$ satisfying (47), there is a 2-dimensional space of solutions for $\gamma$. This leads to two linearly independent eigensolutions, so each eigenvalue occurs with multiplicity 2.

\subsection{Neumann rose graphs}

We can use (47) to derive the condition for $k$ to be an eigenvalue of a rose graph quantised with the Laplace operator and Neumann boundary conditions. The key observation is that if we choose the $\mathrm{SU}(2)$ matrices to be

$$
u_{\mathrm{o}(b)}=u_{\mathrm{t}(b)}=\left(\begin{array}{ll}
1 & 0 \\
0 & 1
\end{array}\right) \quad \text { for all bonds } b,
$$

and choose $m=0$, then the spectral problem given by (4) and (39) degenerates into a pair of independent copies of the Neumann eigenproblem for the Laplace operator.

Since $\tilde{w}_{b}=\mathrm{I}_{2}$ for all $b \in \mathcal{B}$ there will be eigenvalues of the form $k=2 n \pi / L_{b}$ for $n \in \mathbb{Z}$. The rational independence of bond lengths implies that $L_{c} k / 2 \pi \notin \mathbb{Z}$ for any other $c \neq b$, so following the analysis of section 2.1 we get (c.f. equation (46)),

$$
\sum_{\substack{c=1 \\ c \neq b}}^{B} \frac{1}{\sin k L_{c}}\left(2 \mathrm{I}_{2}-2 \cos k L_{c} \mathrm{I}_{2}\right) \boldsymbol{\gamma}=\mathbf{0} .
$$

So $\boldsymbol{\gamma}=\mathbf{0}$, and thence $\mathbf{Q}_{c}=\mathbf{R}_{c}=\mathbf{0}$ for all $c \neq b$.

The remaining points of the spectrum come from the condition (47), which in this situation is expressible as

$$
\sum_{b=1}^{B} \frac{1-\cos k L_{b}}{\sin k L_{b}}=\sum_{b=1}^{B} \tan \left(\frac{k L_{b}}{2}\right)=0 .
$$


The equality here between the left-hand sides comes from the observation that

$$
\frac{1-\cos \varphi}{\sin \varphi}=\tan \left(\frac{\varphi}{2}\right), \quad \text { if } \frac{\varphi}{2 \pi} \notin \mathbb{Z}
$$

What is particularly interesting is that condition (50) is exactly the eigenvalue condition for a Neumann star graph [28] with set of bond lengths $\left\{L_{b} / 2: b \in \mathcal{B}\right\}$.

The spectrum $\left\{k_{n}\right\}$ of the Laplace operator on rose graphs with Neumann boundary conditions consists of points $k=k_{n}$ satisfying (50), together with points of the form $k=2 m \pi / L_{b}$ with $b \in \mathcal{B}$ and $m \in \mathbb{Z}$, which interlace the solutions to (50). Eigenfunctions corresponding to the latter class of eigenvalues are supported on a single bond $b$.

\section{Spectral statistics}

For a Dirac rose graph the eigenvalues are almost-surely the solutions $k$ to the equation

$$
Z(k):=Z(k ; \mathbf{L}, \boldsymbol{\theta}):=\sum_{b=1}^{B} \frac{\cos \theta_{b}-\cos \left(k L_{b}\right)}{\sin \left(k L_{b}\right)}=0 .
$$

Define

$$
z(x, \theta):=\frac{\cos \theta-\cos x}{\sin x},
$$

so that

$$
Z(k)=\sum_{b=1}^{B} z\left(k L_{b}, \theta_{b}\right) .
$$

Let us note an alternative form for the function $z(x, \theta)$. From [36] (equations 1.421.3 and 1.422.3) we have the pole expansions:

$$
\cot z=\frac{1}{z}+2 \sum_{k=1}^{\infty} \frac{z}{z^{2}-k^{2} \pi^{2}}
$$

and

$$
\operatorname{cosec} z=\frac{1}{z}+2 \sum_{k=1}^{\infty} \frac{(-1)^{k} z}{z^{2}-k^{2} \pi^{2}}
$$

Since

$$
\frac{z}{z^{2}-k^{2} \pi^{2}}=\frac{1}{2}\left(\frac{1}{z+k \pi}-\frac{k \pi}{1+k^{2} \pi^{2}}+\frac{1}{z-k \pi}+\frac{k \pi}{1+k^{2} \pi^{2}}\right),
$$

we have

$$
\begin{aligned}
z(x, \theta) & =\cos \theta \operatorname{cosec} x-\cot x \\
& =\sum_{m=-\infty}^{\infty}\left((-1)^{m} \cos \theta-1\right)\left(\frac{1}{x+\pi m}-\frac{m \pi}{1+m^{2} \pi^{2}}\right) .
\end{aligned}
$$

(Because of the regularisation, the series in (58) converges absolutely.)

From this representation, we see that the structure of $Z(k)$ on the real axis is a sequence of poles at the points $k=m \pi / L_{b}$ for $m \in \mathbb{Z}$ and $b=1, \ldots, B$. The only way that poles can become closely-spaced is if poles of $z\left(k L_{b}, \theta_{b}\right)$ become close for different bonds $b$. If 
the set of bond-lengths $\left\{L_{1}, \ldots, L_{B}\right\}$ is linearly independent over $\mathbb{Q}$, then the positions of the poles for different $b$ become independent as $k$ becomes large.

By differentiating (52), we get

$$
Z^{\prime}(k)=\sum_{b=1}^{B} L_{b}\left(\frac{1-\cos \left(k L_{b}\right) \cos \theta_{b}}{\sin ^{2}\left(k L_{b}\right)}\right) \geqslant 0,
$$

so that $Z(k)$ is increasing between poles, and there is a unique zero of $Z(k)$ between each consecutive pair of poles.

These observations mean that spectral statistics of Dirac rose graphs fall into the class considered by Bogomolny et al. in [22]. By averaging over the random phases $\left\{\theta_{b}\right\}_{b \in \mathcal{B}}$, their analysis can be used - with only slight modifications - to derive an expression for the averaged pair-correlation function $R_{2}(x)$ (see equation (142), loc. cit.). In the following two sections we derive an approximation to $R_{2}(x)$ for small $x$, by following a method developed in [22] and other places; and for large $x$, by a different method based on the trace formula (36).

\subsection{Small $x$ behaviour of $R_{2}(x)$}

We shall use the method from [13, 22, 5] (see also [37]) that begins with the observation that statistics of small spacings of zeros of a random meromorphic function, real with poles on the real axis, are approximated by statistics of the zeros of a function with three randomly-distributed poles:

$$
\frac{A_{1}}{k-e_{1}}+\frac{A_{2}}{k-e_{2}}+\frac{A_{3}}{k-e_{3}}=0
$$

where $A_{1}, \ldots, A_{3}$ are random amplitudes, and $e_{1}, \ldots, e_{3}$ are random points. We can assume that the $e_{1}, \ldots, e_{3}$ are distributed over a wide interval of the real axis, since only close spacings will contribute to the approximation for statistics of small zeros spacings.

From (52) and (58), the amplitudes $A_{j}$ are given by the quantities $(-1)^{m_{j}} \cos \theta_{j}-1$ where $m_{j} \in \mathbb{Z}$ and $\theta_{j}$ are independent identically distributed random angles according to (31). More explicitly, $A_{j}$ is a linear transformation of $\cos \theta_{j}$, so it follows from (32) that the probability density of each $A_{j}$ is

$$
\frac{2}{\pi} \sqrt{y(2-y)} \mathbb{1}_{[0,2]}(y) .
$$

We follow the method of [22] which was used for a similar situation in which all $A_{j}$ are equal to 1 . We begin by re-arranging (60), to find that the solutions are given by the quadratic equation:

$$
\left(A_{1}+A_{2}+A_{3}\right) k^{2}-\left(A_{1}\left(e_{2}+e_{3}\right)+A_{2}\left(e_{1}+e_{3}\right)+A_{3}\left(e_{1}+e_{2}\right)\right) k+A_{1} e_{2} e_{3}+A_{2} e_{1} e_{3}+A_{3} e_{1} e_{2}=0 .
$$

Denoting the solutions by $k_{1,2}$, we have

$$
k_{1,2}=\frac{A_{1}\left(e_{2}+e_{3}\right)+A_{2}\left(e_{1}+e_{3}\right)+A_{3}\left(e_{1}+e_{2}\right) \pm \sqrt{\mathcal{D}}}{2\left(A_{1}+A_{2}+A_{3}\right)},
$$

where

$\mathcal{D}=\left(A_{1}\left(e_{2}+e_{3}\right)+A_{2}\left(e_{1}+e_{3}\right)+A_{3}\left(e_{1}+e_{2}\right)\right)^{2}-4\left(A_{1} e_{2} e_{3}+A_{2} e_{1} e_{3}+A_{3} e_{1} e_{2}\right)\left(A_{1}+A_{2}+A_{3}\right)$. 
If $L$ is any real number, then the translation $\left(e_{1}, e_{2}, e_{3}\right) \mapsto\left(e_{1}+L, e_{2}+L, e_{3}+L\right)$ shifts $k_{1}$ and $k_{2}$ by an amount $L$. It is therefore convenient to shift to a set of coördinates in which

$$
A_{1}\left(e_{2}+e_{3}\right)+A_{2}\left(e_{1}+e_{3}\right)+A_{3}\left(e_{1}+e_{2}\right)=0 .
$$

Then (64) becomes

$$
\begin{aligned}
\mathcal{D} & =-4\left(A_{1} e_{2} e_{3}+A_{2} e_{1} e_{3}+A_{3} e_{1} e_{2}\right)\left(A_{1}+A_{2}+A_{3}\right) \\
& =4\left(A_{1}+A_{2}+A_{3}\right)\left(\frac{A_{2}\left(A_{2}+A_{3}\right)}{A_{1}+A_{2}} e_{1}^{2}+\frac{A_{1}\left(A_{1}+A_{3}\right)}{A_{1}+A_{2}} e_{2}^{2}+\frac{2 A_{1} A_{2}}{A_{1}+A_{2}} e_{1} e_{2}\right) .
\end{aligned}
$$

To get the leading contribution to the pair-correlation function, we average over the positions $e_{1}$ and $e_{2}$, and the random amplitudes $A_{1}, \ldots, A_{3}$ :

$$
\mathbb{E}\left(R_{2}(x)\right) \approx \frac{1}{2} \mathbb{E}\left\{\int_{-\infty}^{\infty} \int_{-\infty}^{\infty} \delta(x-\Delta k) \mathrm{d} e_{1} \mathrm{~d} e_{2}\right\},
$$

where

$$
\Delta k=k_{2}-k_{1}=\frac{2 \sqrt{\mathcal{D}}}{2\left(A_{1}+A_{2}+A_{3}\right)},
$$

and $\mathcal{D}$ is given by (66). To perform the integral in (67), we switch to a system of polar coördinates. Let

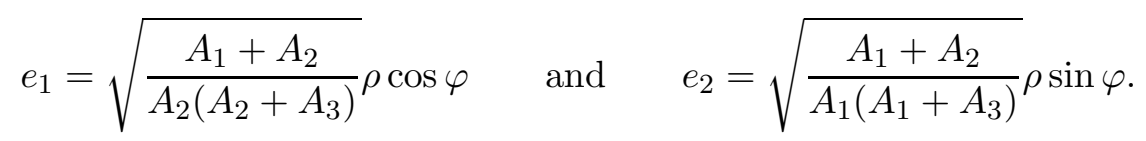

Then we get

$$
\begin{aligned}
\mathbb{E}\left(R_{2}(x)\right) & \approx \frac{1}{2} \mathbb{E}\left\{\int_{0}^{2 \pi} \int_{0}^{\infty} \frac{A_{1}+A_{2}}{\sqrt{A_{1} A_{2}\left(A_{1}+A_{3}\right)\left(A_{2}+A_{3}\right)}} \rho \times\right. \\
& \left.\delta\left(x-\frac{2 \rho}{\sqrt{A_{1}+A_{2}+A_{3}}}\left(1+\frac{2 \sqrt{A_{1} A_{2}}}{\sqrt{\left(A_{1}+A_{3}\right)\left(A_{2}+A_{3}\right)}} \cos \varphi \sin \varphi\right)^{1 / 2}\right) \mathrm{d} \rho \mathrm{d} \varphi\right\},
\end{aligned}
$$

which becomes, after a further substitution,

$$
\begin{aligned}
\mathbb{E}\left(R_{2}(x)\right) \approx & \frac{1}{2} \mathbb{E}\left\{\frac{A_{1}+A_{2}}{\sqrt{A_{1} A_{2}\left(A_{1}+A_{3}\right)\left(A_{2}+A_{3}\right)}} \frac{A_{1}+A_{2}+A_{3}}{4} \times\right. \\
& \left.\int_{0}^{2 \pi}\left(1+\frac{2 \sqrt{A_{1} A_{2}}}{\sqrt{\left(A_{1}+A_{3}\right)\left(A_{2}+A_{3}\right)}} \cos \varphi \sin \varphi\right)^{-1} \mathrm{~d} \varphi \int_{0}^{\infty} \rho \delta(x-\rho) \mathrm{d} \rho\right\} .
\end{aligned}
$$

We use the known integral formula

$$
\int_{0}^{2 \pi} \frac{\mathrm{d} \varphi}{1+a \cos \varphi \sin \varphi}=\frac{2 \pi}{\sqrt{1-a^{2} / 4}}, \quad \text { for }|a|<2,
$$

to get

$$
\begin{aligned}
\int_{0}^{2 \pi}\left(1+\frac{2 \sqrt{A_{1} A_{2}}}{\sqrt{\left(A_{1}+A_{3}\right)\left(A_{2}+A_{3}\right)}} \cos \varphi \sin \varphi\right)^{-1} \mathrm{~d} \varphi & =2 \pi\left(1-\frac{A_{1} A_{2}}{\left(A_{1}+A_{3}\right)\left(A_{2}+A_{3}\right)}\right)^{-1 / 2} \\
& =\frac{2 \pi \sqrt{\left(A_{1}+A_{3}\right)\left(A_{2}+A_{3}\right)}}{A_{3}^{1 / 2} \sqrt{A_{1}+A_{2}+A_{3}}}
\end{aligned}
$$


Substituting into (71) we arrive to

$$
\mathbb{E}\left(R_{2}(x)\right) \approx \frac{\pi x}{4} \mathbb{E}\left\{\frac{\left(A_{1}+A_{2}\right) \sqrt{A_{1}+A_{2}+A_{3}}}{\sqrt{A_{1} A_{2} A_{3}}}\right\} .
$$

As we are assuming that the $A_{j}$ are identically distributed, we may symmetrize (74) and get

$$
\mathbb{E}\left(R_{2}(x)\right) \approx \frac{\pi x}{6} \mathbb{E}\left\{\frac{\left(A_{1}+A_{2}+A_{3}\right)^{3 / 2}}{\sqrt{A_{1} A_{2} A_{3}}}\right\} .
$$

This coïncides with equation (155) from [22], where it was stated but not explicitly derived.

We have to evaluate (75) in the case that $A_{1}, \ldots, A_{3}$ are independent and identically distributed with probability density (61). To do that, it is helpful to observe that since the $A_{j}$ are identically distributed, we have

$$
\begin{aligned}
\mathbb{E}\left\{\frac{\left(A_{1}+A_{2}+A_{3}\right)^{3 / 2}}{\sqrt{A_{1} A_{2} A_{3}}}\right\}=3 \mathbb{E}\left\{\frac{A_{1}^{3 / 2}}{A_{2}^{1 / 2} A_{3}^{1 / 2}} \frac{1}{\sqrt{A_{1}+A_{2}+A_{3}}}\right\} \\
+6 \mathbb{E}\left\{\frac{A_{1}^{1 / 2} A_{2}^{1 / 2}}{A_{3}^{1 / 2}} \frac{1}{\sqrt{A_{1}+A_{2}+A_{3}}}\right\} .
\end{aligned}
$$

Also, since $A_{j}>0$ almost-surely, we also have the identity

$$
\frac{1}{\sqrt{A_{1}+A_{2}+A_{3}}}=\frac{1}{\sqrt{\pi}} \int_{-\infty}^{\infty} \mathrm{e}^{-\left(A_{1}+A_{2}+A_{3}\right) x^{2}} \mathrm{~d} x .
$$

By Fubini's theorem, we have, from (76),

$$
\begin{aligned}
& \mathbb{E}\left\{\frac{\left(A_{1}+A_{2}+A_{3}\right)^{3 / 2}}{\sqrt{A_{1} A_{2} A_{3}}}\right\}=\frac{3}{\sqrt{\pi}} \int_{-\infty}^{\infty} \mathbb{E}\left\{A_{1}^{3 / 2} A_{2}^{-1 / 2} A_{3}^{-1 / 2} \mathrm{e}^{-\left(A_{1}+A_{2}+A_{3}\right) x^{2}}\right\} \mathrm{d} x \\
& +\frac{6}{\sqrt{\pi}} \int_{-\infty}^{\infty} \mathbb{E}\left\{A_{1}^{1 / 2} A_{2}^{1 / 2} A_{3}^{-1 / 2} \mathrm{e}^{-\left(A_{1}+A_{2}+A_{3}\right) x^{2}}\right\} \mathrm{d} x \\
& =\frac{3}{\sqrt{\pi}} \int_{-\infty}^{\infty} I_{3 / 2}(x) I_{-1 / 2}(x)^{2}+2 I_{1 / 2}(x)^{2} I_{-1 / 2}(x) \mathrm{d} x,
\end{aligned}
$$

where

$$
I_{\nu}(x):=\mathbb{E}\left\{A_{1}^{\nu} \mathrm{e}^{-A_{1} x^{2}}\right\}=\frac{2}{\pi} \int_{0}^{2} y^{\nu+1 / 2}(2-y)^{1 / 2} \mathrm{e}^{-y x^{2}} \mathrm{~d} y .
$$

From [36] formula 3.383.1, we find that

$$
I_{\nu}(x)=\frac{2^{\nu+2}}{\sqrt{\pi}} \frac{\Gamma(\nu+3 / 2)}{\Gamma(\nu+3)}{ }_{1} F_{1}\left(\nu+\frac{3}{2} ; \nu+3 ;-2 x^{2}\right) .
$$

The asymptotic behaviour of the confluent hypergeometric function allows us to conclude that

$$
I_{3 / 2}(x) I_{-1 / 2}(x)^{2}+2 I_{1 / 2}(x)^{2} I_{-1 / 2}(x)=\mathrm{O}\left(\frac{1}{x^{10}}\right) \quad \text { as } x \rightarrow \pm \infty,
$$

so that the integral in (78) converges quickly, and may be accurately evaluated using numerical integration techniques. So doing, we arrive at the following result:

$$
\mathbb{E}\left(R_{2}(x)\right) \approx \frac{\pi c}{6} x \quad \text { as } x \rightarrow 0,
$$


where

$$
c \approx 6.781 \ldots
$$

The numerical value for the slope in (82) is approximately $3.550 \ldots$, which is slightly larger than the corresponding slope for star graphs quantised with the Laplace operator (2).

In order to verify the calculation of the constant $c$ we have performed a Monte-Carlo simulation. By randomly generating $10^{6}$ realisations of the random variable

$$
\frac{\left(A_{1}+A_{2}+A_{3}\right)^{3 / 2}}{\sqrt{A_{1} A_{2} A_{3}}}
$$

we found a sample average of 6.785 with a standard deviation of mean of $3.296 \times 10^{-3}$.

\subsection{Large $x$ behaviour of $R_{2}(x)$}

We base our study of the pair-correlation function at large $x$ on the trace formula. Starting from a smoothed version of (1) and applying a standard argument, using the trace formula, we get that

$$
\begin{aligned}
\lim _{N \rightarrow \infty} \frac{1}{4 N} \sum_{m=0}^{N} & \sum_{n=0}^{N} g\left(\lambda_{n}-\lambda_{m}\right) \\
& =\hat{g}(0)+\frac{1}{\mathscr{L}^{2}} \sum_{p, q \in \mathcal{P}} \frac{A_{p} A_{q} \ell_{p} \ell_{q}}{r_{p} r_{q}}\left(\frac{\operatorname{Tr} d_{p}}{2} \frac{\operatorname{Tr} d_{q}}{2}\right) \delta_{\ell_{p}, \ell_{q}}\left(\hat{g}\left(\frac{\ell_{p}}{\mathscr{L}}\right)+\hat{g}\left(\frac{-\ell_{p}}{\mathscr{L}}\right)\right) \\
& =\hat{g}(0)+\int_{-\infty}^{\infty} \hat{g}(\tau) K_{2}(\tau) \mathrm{d} \tau
\end{aligned}
$$

where $K_{2}(\tau)$ is the distribution defined by

$$
K_{2}(\tau):=\frac{1}{\mathscr{L}^{2}} \sum_{p, q \in \mathcal{P}} \frac{A_{p} A_{q} \ell_{p} \ell_{q}}{r_{p} r_{q}}\left(\frac{\operatorname{Tr} d_{p}}{2} \frac{\operatorname{Tr} d_{q}}{2}\right) \delta\left(|\tau|-\frac{\ell_{p}}{\mathscr{L}}\right) \delta_{\ell_{p}, \ell_{q}} .
$$

The quantity (86) is sometimes called the pair-correlation form factor and its behaviour as $\tau \downarrow 0$ will determine the large $x$ behaviour of $R_{2}(x)$.

The leading-order behaviour of the form factor can be determined using Berry's diagonal argument [38], and was investigated for generic graphs quantised with the Dirac operator in [30].

Let us denote by $\mathfrak{L}$ the set of all possible lengths of periodic orbits. Orbits with exactly the same length are said to belong to the same degeneracy class. We sort the sums in (86) by degeneracy class:

$$
K_{2}(\tau)=\frac{1}{\mathscr{L}^{2}} \sum_{\ell \in \mathfrak{L}} \ell^{2} \delta\left(|\tau|-\frac{\ell}{\mathscr{L}}\right)\left(\sum_{\substack{p \in \mathcal{P} \\ \ell_{p}=\ell}} \frac{A_{p}}{r_{p}} \frac{\operatorname{Tr} d_{p}}{2}\right)^{2} .
$$

If we define

$$
\tilde{K}(t, B):=\frac{2 B}{\mathscr{L}^{2}} \sum_{\ell \in \mathfrak{L}} \ell^{2}\left(\sum_{\substack{p \in \mathcal{P}_{t} \\ \ell_{p}=\ell}} \frac{A_{p}}{r_{p}} \frac{\operatorname{Tr} d_{p}}{2}\right)^{2},
$$

where $\mathcal{P}_{t}$ is the set of periodic orbits of topological length $t$ steps, then $\tilde{K}(t, B) \rightarrow K_{2}(\tau)$ weakly as $B \rightarrow \infty$ provided that $t / 2 B \rightarrow \tau$ as $B \rightarrow \infty$. (One proves this by integrating 
(88) and (87) against a test function localised at $\tau$ and noting that the bounds that we imposed on the bond lengths imply that the delta functions corresponding to orbits with different topological lengths are supported on disjoint intervals.)

Let us order the sum over $\ell$ according to the number of distinct bonds to which the sum is confined, and average with respect to the random spin matrices. Then

$$
\mathbb{E}\left(K_{2}(\tau)\right)=\lim _{\substack{B \rightarrow \infty \\ t / 2 B \rightarrow \tau}} \sum_{j=1}^{B} \tilde{K}_{j}(t, B),
$$

where

$$
\tilde{K}_{j}(t, B):=\frac{2 B}{\mathscr{L}^{2}} \sum_{\substack{\ell \text { restricted } \\ \text { to } j \text { bonds }}} \ell^{2} \mathbb{E}\left(\sum_{\substack{p \in \mathcal{P}_{t} \\ \ell_{p}=\ell}} \frac{A_{p}}{r_{p}} \frac{\operatorname{Tr} d_{p}}{2}\right)^{2},
$$

Our approximation to the averaged form factor will be based on selecting from the sums in (90) only those orbits which back-scatter the maximum number of times in their degeneracy class. As back-scattering is increasingly more strongly weighted as $B \rightarrow \infty$ for a rose graph, this may be expected to give a good approximation to the form factor. This type of approximation was considered for Neumann star graphs in [27], where it was compared to an exact expansion of the form factor, and was shown to reproduce exactly the first four terms of the Maclaurin expansion of the form factor.

Let us first consider the special case $j=1$ of periodic orbits confined to a single bond $b$ of the graph. We will (unlike in [27]) need also to consider the parity of $t$.

In the case that $t$ is even, we can back-scatter $t$ times by bouncing ${ }^{1}$ back-and-forth along a single bond, so the contributing orbits are $t / 2$ repetitions of $b \bar{b}$ (denoting by $\bar{b}$ the reversal of $b$ ) with $B$ choices for the bond $b$. For such an orbit $p$ the repetition number is $r_{p}=t / 2$, the stability amplitude is $A_{p}=(1-1 / B)^{t}$ and $d_{p}=\mathrm{I}_{2}$. Approximating the length of the orbit by $t$ (as each bond length approaches 1 as $B \rightarrow \infty$ ) we get

$$
\lim _{\substack{B \rightarrow \infty \\ t / 2 B \rightarrow \tau}} \tilde{K}_{1}(t, B) \approx \lim _{\substack{B \rightarrow \infty \\ t / 2 B \rightarrow \tau}} B \frac{2 B}{\mathscr{L}^{2}} t^{2}\left(1-\frac{1}{B}\right)^{2 t} \frac{4}{t^{2}}\left(\frac{2}{2}\right)^{2} .
$$

As $\mathscr{L} \rightarrow 2 B$ and $t \sim 2 B \tau$, we get

$$
\begin{aligned}
\lim _{\substack{B \rightarrow \infty \\
t / 2 B \rightarrow \tau}} \tilde{K}_{1}(t, B) & \approx \lim _{B \rightarrow \infty} 2\left(1-\frac{1}{B}\right)^{4 B \tau} \\
& =2 \mathrm{e}^{-4 \tau},
\end{aligned}
$$

for $t$ even.

For $t$ odd, we can back-scatter $t-1$ times by bouncing back-and-forth and the final bond passed over is the same as the penultimate one; for each of $B$ bonds, there are two orbits with maximal back-scattering,

$$
b \bar{b} b \bar{b} \cdots b \bar{b} \bar{b} \quad \text { and } \quad \bar{b} b \bar{b} b \cdots \bar{b} b b .
$$

For these two orbits, respectively $\operatorname{Tr} d_{p}=\operatorname{Tr} w_{b}$ and $\operatorname{Tr} d_{p}=\operatorname{Tr} w_{\bar{b}}=\operatorname{Tr} w_{b}$, referring to (35) and (29). Both orbits have

$$
A_{p}=\left(\frac{1}{B}-1\right)^{t-1} \frac{1}{B}
$$

\footnotetext{
${ }^{1}$ We will refer to a transition of the form $b \bar{b}$ or $\bar{b} b$ as a bounce.
} 
and $\ell_{p} \sim t$ and $r_{p}=1$. Therefore, for $t$ odd, we have

$$
\begin{aligned}
\lim _{\substack{B \rightarrow \infty \\
t / 2 B \rightarrow \tau}} \tilde{K}_{1}(t, B) & \approx \lim _{\substack{B \rightarrow \infty \\
t / 2 B \rightarrow \tau}} B \frac{2 B}{\mathscr{L}^{2}} t^{2}\left(1-\frac{1}{B}\right)^{2 t-2} \frac{1}{B^{2}} \frac{1}{4} \mathbb{E}\left(\left(\operatorname{Tr} w_{\bar{b}}+\operatorname{Tr} w_{b}\right)^{2}\right) \\
& =\frac{1}{8} \lim _{B \rightarrow \infty}\left(1-\frac{1}{B}\right)^{4 \tau B-2} 4 \tau^{2} \mathbb{E}\left(4\left(\operatorname{Tr} w_{b}\right)^{2}\right) \\
& =2 \mathbb{E}\left(\operatorname{Tr} w_{b}\right)^{2} \tau^{2} \mathrm{e}^{-4 \tau} .
\end{aligned}
$$

For a random matrix $w \in \mathrm{SU}(2)$ with Haar measure, $\mathbb{E}(\operatorname{Tr} w)^{2}=1$, so we get

$$
\lim _{\substack{B \rightarrow \infty \\ t / 2 B \rightarrow \tau}} \tilde{K}_{1}(t, B) \approx 2 \tau^{2} \mathrm{e}^{-4 \tau} .
$$

Since odd and even values of $t$ have relative density $1 / 2$, we find the total contribution for orbits confined to a single bond is the average of the two cases in (96) and (92):

$$
\left(1+\tau^{2}\right) \mathrm{e}^{-4 \tau} .
$$

We next will consider the case $j=2$. This is more typical of the general case. We now consider orbits confined to two bonds, which we will denote by $a$ and $b$. To maximise back-scattering, we bounce $t_{1}$ times on bond $a$ and $t_{2}$ times on bond $b$, so that $t_{1}+t_{2}=t$. In this way we can achieve $t-2$ back-scatterings (since two transitions are necessarily between different bonds).

For $t_{1}$ and $t_{2}$ there are four possibilities:-

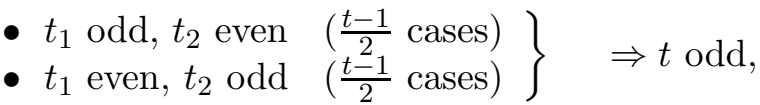

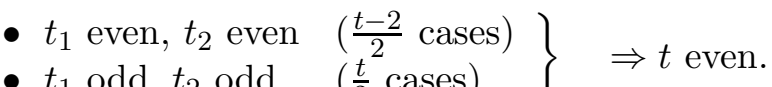

Since odd and even values of $t$ have relative density $1 / 2$, each possibility is weighted approximately $t / 4$ in its contribution to the form factor as $t$ becomes large.

In the first of the four possibilities listed above there are four members of the degeneracy class, which we may label as:

$$
\begin{aligned}
& a \bar{a} a \bar{a} \cdots a \bar{a} a b \bar{b} \cdots b \bar{b} \\
& \bar{a} a \bar{a} a \cdots \bar{a} a \bar{a} b \bar{b} \cdots b \bar{b} \\
& a \bar{a} a \bar{a} \cdots a \bar{a} a \bar{b} b \cdots \bar{b} b \\
& \bar{a} a \bar{a} a \cdots \bar{a} a \bar{a} \bar{b} b \cdots \bar{b} b
\end{aligned}
$$

The values of $\operatorname{Tr} d_{p}$ corresponding to these four orbits reduce to (respectively):

$$
\begin{aligned}
& \operatorname{Tr} w_{a}, \\
& \operatorname{Tr} w_{\bar{a}}=\operatorname{Tr} w_{a}, \\
& \operatorname{Tr} w_{a}, \\
& \operatorname{Tr} w_{\bar{a}}=\operatorname{Tr} w_{a} .
\end{aligned}
$$

Similarly, to the second of the four possibilities, the we find four members of the degeneracy class, each with $\operatorname{Tr} d_{p}=\operatorname{Tr} w_{b}$. 
In the third possibilities, each bond is paired with its reversal an equal number of times, and the four members of the degeneracy class have $\operatorname{Tr} d_{p}=\operatorname{Tr} \mathrm{I}_{2}=2$.

Finally, for the final possibility, the values of $\operatorname{Tr} d_{p}$ are (respectively):

$$
\begin{aligned}
& \operatorname{Tr} w_{a} w_{b}, \\
& \operatorname{Tr} w_{\bar{a}} w_{b}, \\
& \operatorname{Tr} w_{a} w_{\bar{b}}, \\
& \operatorname{Tr} w_{\bar{a}} w_{\bar{b}} .
\end{aligned}
$$

This careful categorisation of each case reveals that the value of number of $\mathrm{SU}(2)$ matrices appearing in the expressions for $\operatorname{Tr} d_{p}$ depends only on the number of odd values of $k_{j}$.

In all cases, we have:

$$
\begin{aligned}
A_{p} & =\left(\frac{1}{B}-1\right)^{t-2} \frac{1}{B^{2}} \\
\ell_{p} & \sim t \\
r_{p} & =1
\end{aligned}
$$

and there are $\frac{B(B-1)}{2} \sim \frac{B^{2}}{2}$ choices for the pair $(a, b)$, since the $B(B-1)$ free choices of bonds would lead to cyclic permutations, which should be counted only once in the trace formula.

Putting the ingredients together, we have

$$
\begin{gathered}
\lim _{\substack{B \rightarrow \infty \\
t / 2 B \rightarrow \tau}} \tilde{K}_{2}(t, B) \approx \lim _{\substack{B \rightarrow \infty \\
t / 2 B \rightarrow \tau}} \\
\frac{B^{2}}{2} \frac{2 B}{\mathscr{L}^{2}} t^{2}\left(1-\frac{1}{B}\right)^{2 t-2} \frac{1}{B^{4}} \frac{t}{4} \frac{1}{4}\left(\mathbb{E}\left(4^{2}\left(\operatorname{Tr} w_{a}\right)^{2}\right)+\mathbb{E}\left(4^{2}\left(\operatorname{Tr} w_{b}\right)^{2}\right)\right. \\
\left.+8^{2}+\mathbb{E}\left(\left(\operatorname{Tr} w_{a} w_{b}+w_{\bar{a}} w_{b}+w_{a} w_{\bar{b}}+w_{\bar{a}} w_{\bar{b}}\right)^{2}\right)\right) .
\end{gathered}
$$

As before, $\mathbb{E}\left(\left(\operatorname{Tr} w_{a}\right)^{2}\right)=\mathbb{E}\left(\left(\operatorname{Tr} w_{b}\right)^{2}\right)=1$, and we find that

$$
\mathbb{E}\left(\left(\operatorname{Tr} w_{a} w_{b}+w_{\bar{a}} w_{b}+w_{a} w_{\bar{b}}+w_{\bar{a}} w_{\bar{b}}\right)^{2}\right)=4,
$$

as a special case of equation (111) below. Therefore,

$$
\begin{aligned}
\lim _{\substack{B \rightarrow \infty \\
t / 2 B \rightarrow \tau}} \tilde{K}_{2}(t, B) & \approx \lim _{B \rightarrow \infty} \frac{8 \tau^{3}}{64}\left(1-\frac{1}{B}\right)^{4 \tau B}(16+16+64+4) \\
& =\frac{25}{2} \tau^{3} \mathrm{e}^{-4 \tau} .
\end{aligned}
$$

We now turn to the general case of orbits confined to $j$ bonds. An orbit with maximal back-scattering will bounce along bond $a_{\ell}$ a total of $t_{\ell}$ times, $\ell=1, \ldots, j$, in such a way that

$$
t_{1}+\cdots+t_{j}=t
$$

In this way, $t-j$ back-scatterings are achieved.

The weighting that each possibility receives in its contribution to the form factor has two components. The first component is based on the relative density of occurrences of odd or even $t_{\ell}$ s. Since there are are $2^{j}$ possible choices for $t_{\ell}$ to be odd or even, this factor 
is simply $1 / 2^{j}$. A second factor comes from the number of ways to decompose $t$ in the form (106). The number of such decompositions is the number of ways to choose $j-1$ numbers (the transition points: $t_{1}, t_{1}+t_{2}$, et cetera) from a total of $t-1$ possible ones. Although these two factors are not independent, since the odd and even choices for $t_{\ell}$ are evenly distributed, the weight of each possibility is approximately their product:

$$
\frac{1}{2^{j}}\left(\begin{array}{c}
t-1 \\
j-1
\end{array}\right) \sim \frac{t^{j-1}}{2^{j}(j-1) !} .
$$

Within each possibility it is the number of odd $t_{j}$ s that determines the value of the trace factor; after cancellations we are left with

$$
\operatorname{Tr} d_{p}=\operatorname{Tr}\left(w_{a_{i_{1}}}^{\alpha_{i_{1}}} \cdots w_{a_{i_{r}}}^{\alpha_{i_{r}}}\right),
$$

where there are precisely $r$ indices $\left\{i_{1}, \ldots, i_{r}\right\}$ for which $t_{i_{\ell}}$ is odd, and each $\alpha_{i_{\ell}}= \pm 1$. There are $2^{j}$ members of each degeneracy class, and $2^{r}$ ways that $\alpha_{i_{\ell}}= \pm 1$. So we will need to calculate

$$
\mathbb{E}\left\{\left(2^{j-r} \sum_{\alpha_{i_{\ell}}= \pm 1} \operatorname{Tr}\left(w_{a_{i_{1}}}^{\alpha_{i_{1}}} \cdots w_{a_{i_{r}}}^{\alpha_{i_{r}}}\right)\right)^{2}\right\} .
$$

Since every combination of $\alpha_{i_{\ell}}= \pm 1$ occurs in the sum, and $w_{a_{i_{\ell}}}+w_{a_{i_{\ell}}}^{-1}=\operatorname{Tr}\left(w_{a_{i_{\ell}}}\right) \mathrm{I}_{2}$ we have

$$
\sum_{\alpha_{i_{\ell}}= \pm 1} w_{a_{i_{1}}}^{\alpha_{i_{1}}} \cdots w_{a_{i_{r}}}^{\alpha_{i_{r}}}=\left(\operatorname{Tr} w_{a_{1}}\right) \cdots\left(\operatorname{Tr} w_{a_{r}}\right) \mathrm{I}_{2}
$$

Thus,

$$
\begin{aligned}
\mathbb{E}\left\{\left(2^{j-r} \sum_{\alpha_{i_{\ell}}= \pm 1} \operatorname{Tr}\left(w_{a_{i_{1}}}^{\alpha_{i_{1}}} \cdots w_{a_{i_{r}}}^{\alpha_{i_{r}}}\right)\right)^{2}\right\} & =4^{j+1-r} \mathbb{E}\left\{\left(\operatorname{Tr} w_{a_{1}}\right)^{2} \cdots\left(\operatorname{Tr} w_{a_{r}}\right)^{2}\right\} \\
& =4^{j+1-r} .
\end{aligned}
$$

Finally, we note that there are $\left(\begin{array}{l}j \\ r\end{array}\right)$ combinations of ways that there can be $r$ odd indices out of a total of $j$.

In all cases, we have:

$$
\begin{aligned}
A_{p} & =\left(\frac{1}{B}-1\right)^{t-j} \frac{1}{B^{j}} \\
\ell_{p} & \sim t \quad \text { and } \\
r_{p} & =1 .
\end{aligned}
$$

The number of choices for the $j$ bonds on which the orbits are confined is $\frac{1}{j} \frac{B !}{(B-j) !} \sim \frac{B^{j}}{j}$ (the factor $1 / j$ is to account for cyclic invariance). We get

$$
\begin{aligned}
\lim _{\substack{B \rightarrow \infty \\
t / 2 B \rightarrow \tau}} \tilde{K}_{j}(t, B) & =\lim _{\substack{B \rightarrow \infty \\
t / 2 B \rightarrow \tau}} \frac{B^{j}}{j} \frac{2 B}{\mathscr{L}^{2}} t^{2}\left(1-\frac{1}{B}\right)^{2 t-2 j} \frac{1}{B^{2 j}} \frac{t^{j-1}}{2^{j}} \frac{1}{(j-1) !} \frac{1}{4}\left\{\sum_{j=0}^{r}\left(\begin{array}{l}
j \\
r
\end{array}\right) 4^{j+1-r}\right\} \\
& =\lim _{\substack{B \rightarrow \infty \\
t / 2 B \rightarrow \tau}} \frac{t^{j+1}}{2^{j+1} B^{j+1}}\left(1-\frac{1}{B}\right)^{2 t-2 j} \frac{1}{j !}\left\{\sum_{j=0}^{r}\left(\begin{array}{l}
j \\
r
\end{array}\right) 4^{j-r}\right\} \\
& =\tau^{j+1} \mathrm{e}^{-4 \tau} \frac{5^{j}}{j !} .
\end{aligned}
$$


We remark that upon substituting $j=2$ into (115) we recover (105), as expected.

Substituting (97) and (115) into (89) we get

$$
\begin{aligned}
\mathbb{E}\left(K_{2}(\tau)\right) & \approx\left(1+\tau^{2}\right) \mathrm{e}^{-4 \tau}+\sum_{j=2}^{\infty} \tau^{j+1} \mathrm{e}^{-4 \tau} \frac{5^{j}}{j !} \\
& =\left(1+\tau^{2}\right) \mathrm{e}^{-4 \tau}+\tau \mathrm{e}^{-4 \tau}\left(\mathrm{e}^{5 \tau}-1-5 \tau\right) \\
& =\left(1-\tau-4 \tau^{2}\right) \mathrm{e}^{-4 \tau}+\tau \mathrm{e}^{\tau} .
\end{aligned}
$$

Expanding (116) as a Maclaurin series, we get

$$
\mathbb{E}\left(K_{2}(\tau)\right) \approx 1-4 \tau+9 \tau^{2}-\frac{13}{6} \tau^{3}+\mathrm{O}\left(\tau^{4}\right),
$$

for small values of $\tau$.

It follows from the fact that the form-factor and the pair-correlation function are related via the Fourier transform that the small $\tau$ asymptotics (116) determine the large $x$ behaviour of the averaged pair-correlation function. Namely, we have [39, page 102] that if $k(\tau)$ is even and

$$
k(\tau) \sim 1+\sum_{k=1}^{\infty} a_{k} \tau^{k}
$$

and

$$
1-k(\tau)=\int_{-\infty}^{\infty}(1-r(x)) \mathrm{e}^{2 \pi \mathrm{i} x \tau} \mathrm{d} x,
$$

then

$$
r(x) \sim 1+2 \mathfrak{R e}\left\{\sum_{k=1}^{\infty}\left(\frac{-\mathrm{i}}{2 \pi}\right)^{k+1} \frac{a_{k} k !}{x^{k+1}}\right\} .
$$

Applying this to the form-factor approximation (117), we get the following approximation to the pair-correlation function:

$$
\mathbb{E}\left(R_{2}(x)\right) \approx 1+\frac{2}{\pi^{2} x^{2}}-\frac{13}{8 \pi^{4} x^{4}}+\mathrm{O}\left(\frac{1}{x^{6}}\right),
$$

for large values of $x$.

\section{Conclusions}

We have investigated the spectral statistics of a quantum graph quantised with the Dirac operator, for which the Schrödinger-operator-quantised counterpart (Laplace operator) has intermediate spectral statistics. The shape of the graph has a single central vertex, and all bonds are connected at both ends to that vertex. We call this graph a rose.

We have shown that the generic condition for $k$ to be an eigenvalue is that it satisfies the non-linear equation

$$
\sum_{b=1}^{B} \frac{\cos \theta_{b}-\cos k L_{b}}{\sin k L_{b}}=0,
$$

where the angles $\theta_{b}$ are determined by a set of $B$ matrices from $\mathrm{SU}(2)$ that rotate the spinor during its passage along the bonds. Each eigenvalue occurs with multiplicity 2 (Kramer's degeneracy). 
We have investigated the behaviour of the spectral pair correlation function $R_{2}(x)$ in the limit $B \rightarrow \infty$ for large and small values of $x$, when it is averaged over random realisations of the $\mathrm{SU}(2)$ matrices chosen with Haar measure.

For small values of $x$ we have found

$$
\mathbb{E}\left(R_{2}(x)\right) \approx \frac{\pi c}{6} x \quad \text { as } x \rightarrow 0,
$$

where $c \approx 6.781 \ldots$

For large values of $x$ we have determined

$$
\mathbb{E}\left(R_{2}(x)\right) \approx 1+\frac{2}{\pi^{2} x^{2}}-\frac{13}{8 \pi^{4} x^{4}}+\mathrm{O}\left(\frac{1}{x^{6}}\right) .
$$

The behaviour of the pair correlation function is different to the pair correlation function for star graphs quantised with the Laplace operator (see also figure 4 below for a numerical comparison). It is too early to conjecture that the behaviour that we found is universal for systems with intermediate statistics and a symplectic symmetry, but this possibility merits further investigation.

It is interesting to note that while we considered elements $w_{b}$ chosen with Haar measure in $\mathrm{SU}(2)$ the large parameter asymptotic of $R_{2}(x)$ would be the same if $w_{b}$ is chosen from any irreducible representation of a subgroup $\Gamma \subset \mathrm{SU}(2)$. The reason for this was seen in (111) where to evaluate the asymptotic it was only necessary to know that $\mathbb{E}\left\{(\operatorname{Tr} w)^{2}\right\}=1$. As $\operatorname{Tr} w$ is the character of an element of the subgroup when the average is carried out over $\Gamma$ for an irreducible representation of $\Gamma$ the result must still be one by the character orthogonality relations. So, for example, choosing the spin transformations $w_{b}$ on the rose from the finite subgroup

$$
\Gamma=\left\{ \pm \mathrm{I}, \pm \mathrm{i} \sigma_{x}, \pm \mathrm{i} \sigma_{y}, \pm \mathrm{i} \sigma_{z}\right\},
$$

where $\sigma_{j}$ is a Pauli matrix, will not change the large $x$ asymptotic of $R_{2}(x)$. The small parameter asymptotic, in contrast, depends on the distribution of the $A_{j}$ given in (61) which will vary if the spin transformations are chosen from an irreducible representation of a subgroup.

\subsection{Some numerics}

In order to numerically verify the small and large $x$ behaviour of the pair correlation function found in the previous section, we performed numerical calculations of the eigenvalues of rose graphs quantised with the Dirac operator, and calculated the empirical pair correlation statistic. To implement the averaging over the random choice of $\mathrm{SU}(2)$ matrices at the graph vertex, we performed the calculations 100 times with random Haar-distributed matrices and averaged the results. In each realisation, 150000 eigenvalues were calculated.

In figure 3 we compare the numerically-calculated pair correlation function with the predictions of (123) and (124), and find a good agreement in the range of validity.

The agreement gets better as the number of bonds increases, as is to be expected since our analytical calculations relate to the limit $B \rightarrow \infty$. This improvement is demonstrated in figure 6 in which a comparison is made between the pair-correlation function for 21, 61 and 101 bond graphs, and the large $x$ prediction of (124). A clear increase in adherence to the prediction is displayed as the number of bonds increases.

One may wonder if averaging over both the random $\mathrm{SU}(2)$ matrices and bond lengths will lead to a different pair-correlation function. Figure 5 compares such a calculated 
empirical curve with that for corresponding graph with averaging over the $\mathrm{SU}(2)$ matrices only, for a $B=101$ bond Dirac rose graph. There is no noticeable difference in the curves so obtained.

It is of interest to compare the pair correlation function for the Neumann star graphs with the Laplace operator, and the Dirac rose graphs. This is done in figure 4 for graphs with 101 bonds in both cases. Qualitatively the curves are similar in appearance, but there is some noticeable difference, which can already be explained analytically around the point $x=0$ (compare (123) and (2)).

\section{Acknowledgements}

We are grateful to Jon Keating for encouraging us to work on this problem, and acknowledge fruitful conversations with Gregory Berkolaiko, and Jens Bolte regarding this work.

JMH would like to thank Bristol University for their hospitality during his sabbatical during which some of the work was carried out. BW has been financially supported by EPSRC grant number EP/H046240/1. JMH was supported by the Baylor University research leave and summer sabbatical programs.

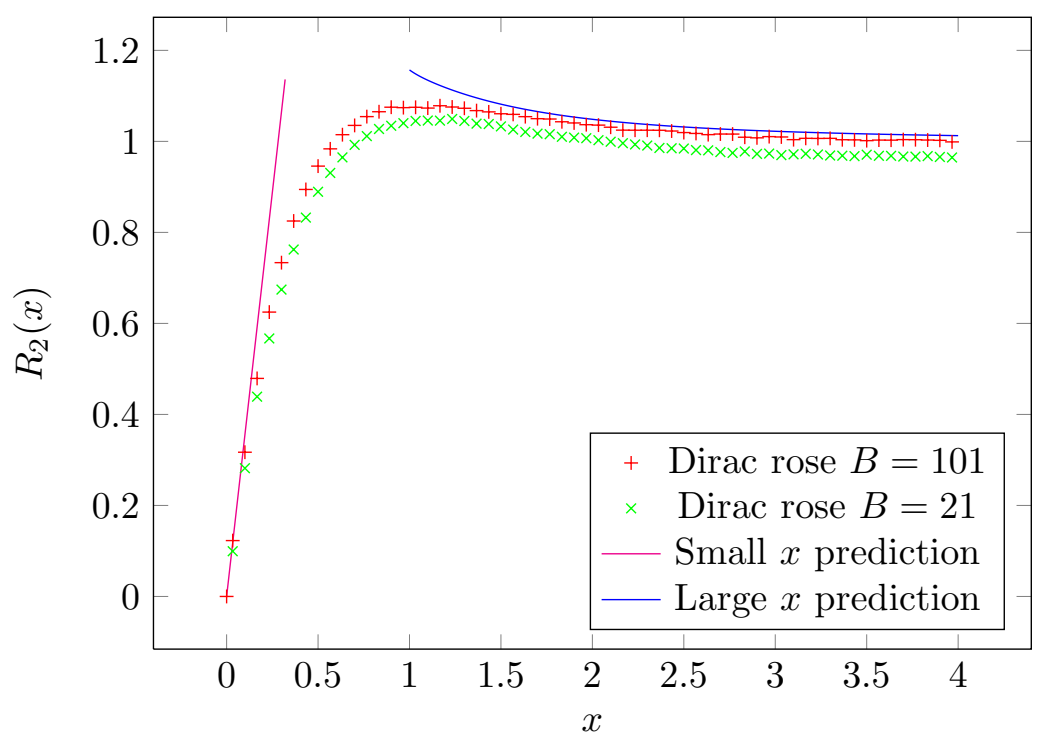

Figure 3: The pair-correlation function numerically calculated for a $B=21$ bond (green) and a $B=101$ bond (red) Dirac rose graph. Also plotted are the curves corresponding to the large and small values of the parameter, (124) and (123) respectively. The numerical plots are averaged over 100 realisations of the SU(2) matrices, and 150000 eigenvalues were computed.

\section{References}

[1] O. Bohigas, M.-J. Giannoni, and C. Schmit (1984) "Characterization of chaotic quantum spectra and universality of level fluctuation laws," Phys. Rev. Lett. 52, pp. 1-4. 


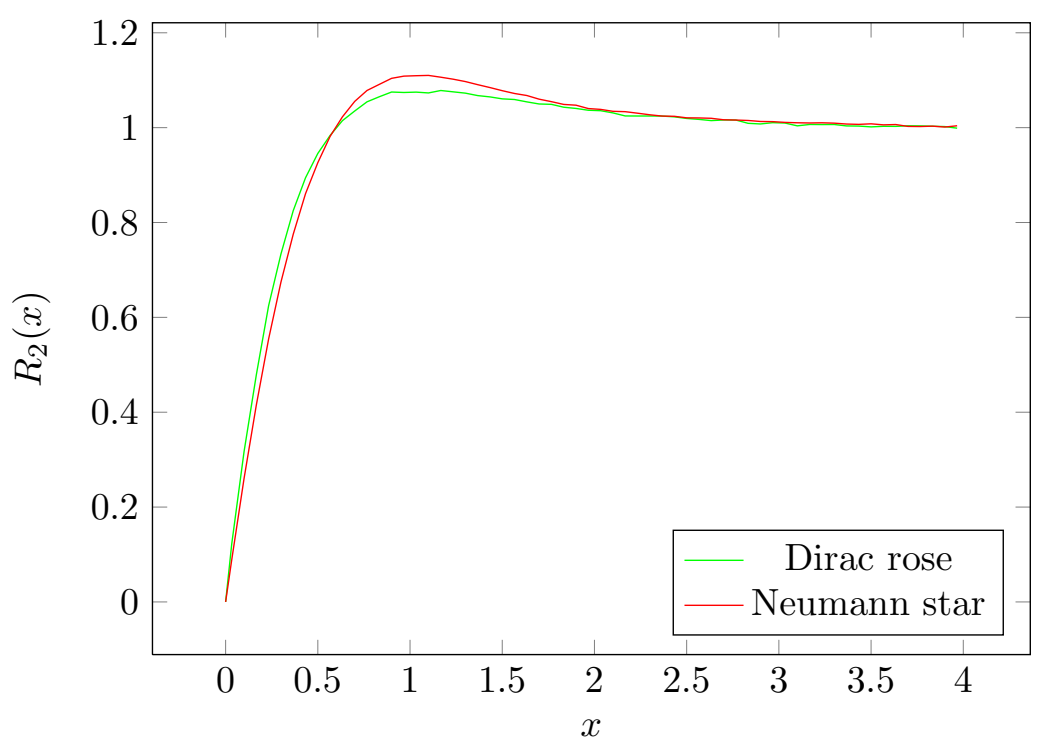

Figure 4: A comparison of the pair-correlation function numerically calculated for a $B=$ 101 bond Neumann star graph (red) and Dirac rose graph (green). The numerical plots are averaged over 100 realisations of the $\mathrm{SU}(2)$ matrices for the rose graph and 100 realisations of the bond lengths for the star graph, and 150000 eigenvalues were computed.

[2] G. Casati, F. Valz-Griz, and I. Guarneri (1980) "On the connection between the quantization of nonintegrable systems and statistical theory of spectra," Lett. Nuovo Cimento 28, pp. 279-282.

[3] R. Scharf, B. Deitz, M. Kuś, F. Haake, and M. V. Berry (1988) "Kramer's degeneracy and quartic level repulsion," Europhys. Lett. 5, pp. 383-389.

[4] E. Caurier and B. Grammaticos (1989) "Extreme level repulsion for chaotic quantum Hamiltonians," Phys. Lett. A 136, pp. 387-390.

[5] T. Gorin, M. Müller, and P. Šeba (2001) "Comment on "models of intermediate spectral statistics'," Phys. Rev. E 63, art. no. 068201.

[6] B. I. Shklovskii, B. Shapiro, B. R. Sears, P. Lambrianides, and H. B. Shore (1993) "Statistics of spectra of disordered systems near the metal-insulator transition," Phys. Rev. B 47, pp. 11487-11490.

[7] G. Date, S. R. Jain, and M. V. N. Murthy (1994) "Rectangular billiard in the presence of a flux line," Phys. Rev. E 51, pp. 198-203.

[8] S. Rahav and S. Fishman (2001) "Spectral statistics of the rectangular billiard with a flux line," Found. Phys. 31, pp. 115-146.

[9] E. Bogomolny, O. Giraud, and C. Schmidt (2001) "Periodic orbit contributions to the 2-point correlation form factor for pseudo-integrable systems," Commun. Math. Phys. 222, pp. 327-369. 


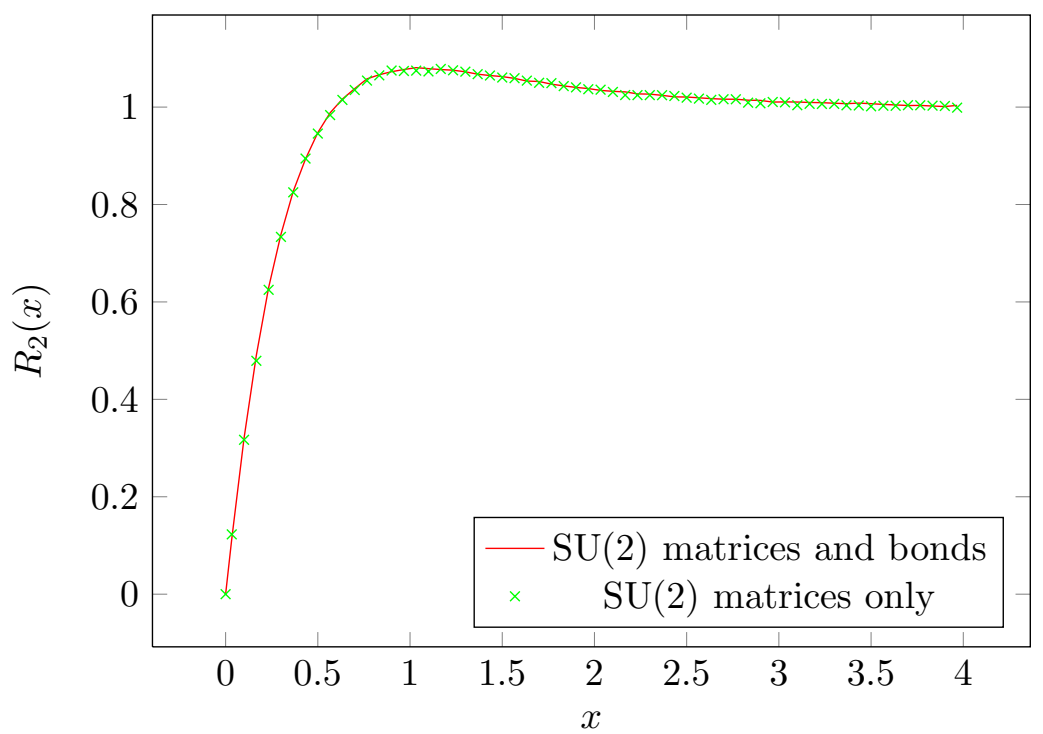

Figure 5: The pair-correlation function numerically calculated for a $B=101$ bond Dirac rose graph (green), 100 realisations of the $\mathrm{SU}(2)$ matrices only (green points) and 100 realisations of the matrices and bond lengths (red curve). 150000 eigenvalues were computed.

[10] P. Šeba (1990) "Wave chaos in singular quantum billiard," Phys. Rev. Lett. 64, pp. $1855-1858$.

[11] P. Šeba and K. Życzkowski (1991) "Wave chaos in quantized clasically nonchaotic systems," Phys. Rev. A 44, pp. 3457-3465.

[12] S. Albeverio and P. Šeba (1991) "Wave chaos in quantum systems with point interaction," J. Stat. Phys. 64, pp. 369-383.

[13] E. B. Bogomolny, U. Gerland, and C. Schmit (1999) "Models of intermediate spectral statistics," Phys. Rev. E 59, pp. R1315-R1318.

[14] B. Grémaud and S. R. Jain (1998) "Spacing distributions for rhombus billiards," $J$. Phys. A 31, pp. L637-L643.

[15] H. D. Parab and S. R. Jain (1996) "Non-universal spectral rigidity of quantum pseudointegrable billiards," J. Phys. A 29, pp. 3903-3910.

[16] T. Tudorovskiy, U. Kuhl, and H.-J. Stöckmann (2010) "Singular statistics revisited," New J. Phys. 12, art. no. 123021.

[17] O. Giraud, J. Marklof, and S. O'Keefe (2004) "Intermediate statistics in quantum maps," J. Phys. A 37, pp. L303-L311.

[18] E. Bogomolny, U. Gerland, and C. Schmit (2001) "Short-range plasma model for intermediate spectral statistics," Eur. Phys. J. B 19, pp. 121-132.

[19] R. L. Weaver and D. Sornette (1995) "Range of spectral correlations in pseudointegrable systems: Gaussian orthogonal ensemble statistics in a rectangular membrane with a point scatterer," Phys. Rev. E 52, pp. 3341-3350. 


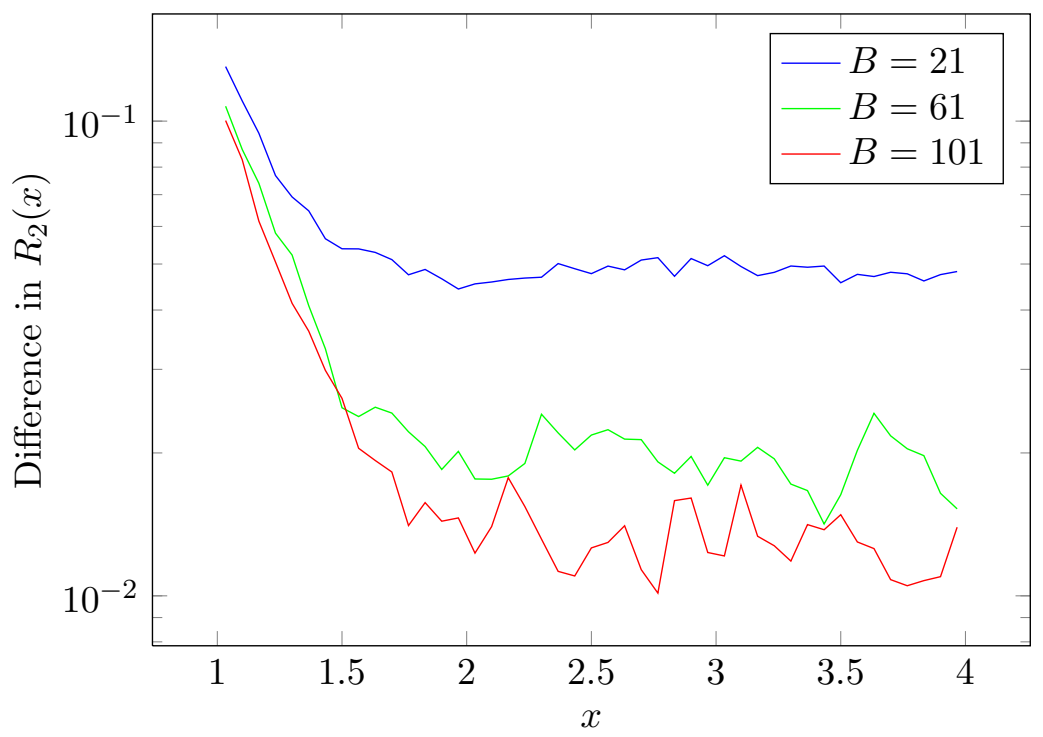

Figure 6: The difference between the asymptotic (124) and the pair-correlation function numerically calculated for a $B=21$ bond (blue), $B=61$ bond (green) and $B=101$ bond (red) Dirac rose graph, plotted on a logarithmic scale.

[20] T. Shigehara, N. Yoshinaga, T. Cheon, and T. Mizusaki (1993) "Level spacing distribution of a singular billiard," Phys. Rev. E 47, pp. R3822-R3825.

[21] E. Bogomolny, P. Leboeuf, and C. Schmit (2000) "Spectral statistics of chaotic systems with a pointlike scatterer," Phys. Rev. E 85, pp. 2486-2489.

[22] E. Bogomolny, U. Gerland, and C. Schmit (2001) "Singular statistics," Phys. Rev. E 63, art. no. 036206.

[23] E. Bogomolny, O. Giraud, and C. Schmit (2002) "Nearest-neighbor distribution for singular billiards," Phys. Rev. E 65, art. no. 056214.

[24] E. Bogomolny and O. Giraud (2002) "Semiclassical calculations of the two-point correlation form factor for diffractive systems," Nonlinearity 15, pp. 993-1018.

[25] S. Rahav and S. Fishman (2002) "Spectral statistics of rectangular billiards with localized perturbations," Nonlinearity 15, pp. 1541-1594.

[26] S. Rahav, O. Richman, and S. Fishman (2003) "Point perturbations of circle billiards," J. Phys. A 36, pp. L529-L536.

[27] G. Berkolaiko and J. P. Keating (1999) "Two-point spectral correlations for star graphs," J. Phys. A 32, pp. 7827-7841.

[28] G. Berkolaiko, E. B. Bogomolny, and J. P. Keating (2001) "Star graphs and Šeba billiards," J. Phys. A 34, pp. 335-350.

[29] O. Bohigas and M.-J. Giannoni "Chaotic motion and random matrix theories," in Mathematical and Computational Methods in Nuclear Physics vol. 209 of Lecture Notes in Physics pp. 1-99 Springer 1984. 
[30] J. Bolte and J. Harrison (2003) "Spectral statistics for the Dirac operator on graphs," J. Phys. A 36, pp. 2747-2769.

[31] J. Bolte and J. Harrison (2003) "The spin contribution to the form factor of quantum graphs," J. Phys. A 36, pp. L433-L440.

[32] M. Harmer (2000) "Hermitian symplectic geometry and extension theory," J. Phys. A 33, pp. 9193-9203.

[33] V. Kostrykin and R. Schrader (1999) "Kirchhoff's rule for quantum wires," J. Phys. A 32, pp. 595-630.

[34] P. Kuchment (2004) "Quantum graphs. I. Some basic structures," Waves Random Media 14, pp. S107-S128. Special section on quantum graphs.

[35] T. Kottos and U. Smilansky (1999) "Periodic orbit theory and spectral statistics for quantum graphs," Ann. Phys. 274, pp. 76-124.

[36] I. S. Gradshteyn and I. M. Ryzhik Table of integrals, series, and products. Elsevier/Academic Press, Amsterdam 7th ed. 2007.

[37] F. Barra and P. Gaspard (2000) "On the level spacing distribution in quantum graphs," J. Stat. Phys. 101, pp. 283-319.

[38] M. V. Berry (1985) "Semiclassical theory of spectral rigidity," Proc. R. Soc. London, Ser. A 400, pp. 229-251.

[39] G. Berkolaiko Quantum Star Graphs and Related Systems. Ph.D. thesis, University of Bristol 2000. 